1

2

3

\title{
1 Localized Delivery of CRISPR/dCas9 via Layer-by-Layer Self-Assembling \\ 2 Peptide Coating on Nanofibers for Neural Tissue Engineering \\ 3
}

4 Kunyu Zhang ${ }^{\text {a, } 1 \text {, Wai Hon Chooi }}{ }^{\text {a, }}{ }^{\text {, }}$, Shuang Liu ${ }^{b}$, Jiah Shin Chin ${ }^{\text {a, c }}$, Aoife Murray ${ }^{\text {, }}$, Dean

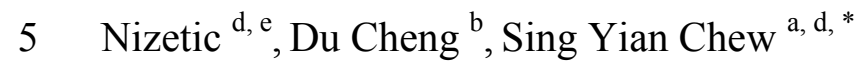

6

$7 \quad{ }^{a}$ School of Chemical \& Biomedical Engineering, Nanyang Technological University, 637459

8 Singapore

$9{ }^{\mathrm{b}}$ Key Laboratory for Polymeric Composite and Functional Materials of Ministry of Education, 10 School of Materials Science and Engineering, Sun Yat-Sen University, Guangzhou 510275, 11 China

$12 \mathrm{c}$ NTU Institute of Health Technologies, Interdisciplinary Graduate School, Nanyang 13 Technological University, Singapore 639798, Singapore

$14{ }^{\mathrm{d}}$ Lee Kong Chian School of Medicine, Nanyang Technological University, 308232 Singapore

$15{ }^{\mathrm{e}}$ The Blizard Institute, Barts \& The London School of Medicine, Queen Mary University of 16 London, 4 Newark St., London E1 2AT, United Kingdom

$18{ }^{1}$ These authors contributed equally to this work. 19

20 * Corresponding author: sychew@ntu.edu.sg (S. Y. Chew) 
2 Keywords: genome editing, gene delivery, CRISPR/Cas9, electrospinning, nerve regeneration

4 Abstract: The clustered regularly interspaced short palindromic repeat (CRISPR) systems have 5 a wide variety of applications besides precise genome editing. In particular, the CRISPR/dCas9

6 system can be used to control specific gene expression by CRISPR activation (CRISPRa) or 7 interference (CRISPRi). However, the safety concerns associated with viral vectors and the 8 possible off-target issues of systemic administration remain huge concerns to be safe delivery 9 methods for CRISPR/Cas9 systems. In this study, a layer-by-layer (LbL) self-assembling peptide 10 (SAP) coating on nanofibers is developed to mediate localized delivery of CRISPR/dCas9 11 systems. Specifically, an amphiphilic negatively charged $\mathrm{SAP}^{-}$is first coated onto PCL 12 nanofibers through strong hydrophobic interactions, and the pDNA complexes and positively 13 charged $\mathrm{SAP}^{+}$-RGD are then absorbed via electrostatic interactions. The SAP-coated scaffolds 14 facilitate efficient loading and sustained release of the pDNA complexes, while enhancing cell 15 adhesion and proliferation. As a proof of concept, the scaffolds are used to activate GDNF 16 expression in mammalian cells, and the secreted GDNF subsequently promotes neurite 17 outgrowth of rat neurons. These promising results suggest that the LbL self-assembling peptide 18 coated nanofibers can be a new route to establish a bioactive interface, which provides a simple 19 and efficient platform for the delivery of CRISPR/dCas9 systems for regenerative medicine. 
2 The clustered regularly interspaced short palindromic repeat (CRISPR) systems have

3 evolved as powerful tools for precise genome editing in diverse biological applications $[1,2]$.

4 These systems use a single guide RNA (sgRNA) to direct the Cas9 nuclease to the target gene,

5 resulting in gene deletion, insertion, and mutation [1-4]. Additionally, a transcriptional regulator

6 can be fused to the dead Cas9 (dCas9), in which the two catalytic domains of Cas9 are

7 inactivated and thus have no cleavage activity [5]. This allows the CRISPR/dCas9 system to be

8 used to activate or interfere with specific gene expression, named CRISPR activation (CRISPRa)

9 and CRISPR interference (CRISPRi) respectively [6, 7]. Unfortunately, apart from these 10 promising features, challenges remained for the CRISPR/Cas9 system to be translated for 11 clinical applications. Although viral vectors like adeno-associated virus (AAV) have shown great 12 efficacy in CRISPR/Cas9 system delivery, the immunogenic response and long-term expression 13 of viral vectors are still the main concerns $[8,9]$. Meanwhile, the uptake of the vectors by 14 undesired tissue or organ, especially during systemic administration, may cause off-target issues, 15 which may lead to tumorigenic or other deleterious events. To overcome these limitations, we 16 sought to develop an effective non-viral approach to deliver CRISPR/Cas9 components in a 17 sustained and localized fashion, which is often required for tissue regeneration.

18 Polycaprolactone (PCL) is widely used for the fabrication of tissue engineering scaffolds by 19 various techniques, including electrospinning and 3D printing [10, 11], owing to its 20 machinability, structural stability, biocompatibility and biodegradability. However, the intrinsic 21 hydrophobicity and absence of reactive ligands on PCL lead to ineffective cell adhesion and 22 biomolecules immobilization, which hinders the further development of the scaffolds for tissue 23 engineering. In addition, the chemical and biological stability of PCL makes it difficult for 
1 surface modification. Lately, inspired by mussel adhesion chemistry, many groups, including

2 ourselves, have demonstrated the easy-to-operate polydopamine coating strategy on PCL

3 scaffolds, which could significantly enhance cell-substrate interactions and biomolecule

4 immobilizations [12-19]. Such bioadhesive coating allowed effective scaffold-mediated delivery

5 of Cas9-sgRNA ribonucleoprotein to cells [18]. However, although the mechanism is still

6 unclear, the special surface chemistry of polydopamine has been reported to be not favorable to

7 some sensitive cells [18]. Therefore, we aim to develop a novel coating strategy that is more

8 cytocompatible and allows scaffold surface-mediated delivery of CRISPR/dCas9 system for

9 CRISPRa. We delivered the systems through dCas9 plasmid DNA (pDNA) in this study due to 10 its low cost, flexibility, and stability, which is useful for us to test on the new coating platform 11 [20]. As a proof of concept, scaffold-mediated gene activation of Glial cell derived neurotrophic 12 factor (GDNF) would be an interesting strategy to stimulate nerve regeneration as it delivers both 13 topographical and biochemical cues to guide cellular and tissue regrowth.

14 In this study, we successfully fashioned a layer-by-layer (LbL) self-assembling peptide 15 (SAP) coating on nanofibers that can mediate localized delivery of CRISPR/dCas9 system to 16 promote neural regeneration. We first established an easy-to-operate approach for the bioactive 17 functionalization of PCL nanofibers by SAP. These SAP-coated nanofibers exhibited good 18 affinity towards pDNA/PEIpro complexes and hence could facilitate the efficient loading and 19 sustained release of such complexes. Owing to the incorporation of RGD peptides, the scaffolds 20 could also support cell adhesion and proliferation. Other than the RGD, our SAP-based scaffold 21 design also allows facile incorporation of other bioactive peptides, thereby offering a superb 22 platform for the investigation of cell-substrate interactions. Furthermore, due to these promising 23 features, the scaffolds were capable of localized delivery of CRISPR/dCas9 system to activate 
1 GDNF expression in mammalian cells, and the secreted GDNF could subsequently promote

2 neurite outgrowth. We believe that the strategy reported in this work opens up a new route to

3 establish a bioactive interface and a simple and efficient platform for the delivery of

4 CRISPR/dCas9 systems for regenerative medicine.

5

\section{2. Materials and methods}

\section{2.1. Materials}

8 Polycaprolactone (average $\mathrm{Mw}=80000)$, 2,2,2-trifluoroethanol (TFE), heparin sodium salt and

9 Triton-X 100 were purchased from Sigma-Aldrich (USA). Sodium hydroxide was purchased

10 from Merck (Germany). All chemicals were used as received without further purification. The 11 self-assembling peptide, SAP $^{-}$(FEFEFEFE) and SAP ${ }^{+}$-RGD (FKFKFKFKGGRGDSP), were 12 synthesized by Genscript (USA) and characterized by HPLC and ESI-MS (Supplementary 13 Methods Section 1. Characterization of SAP). Paraformaldehyde (PFA, 4\%) was purchased from 14 Santa Cruz Biotechnology. PEIpro transfection reagent was purchased from Polyplus (USA). 15 Plasmid pSpCas9(BB)-2A-GFP (PX458) (“Cas9-GFP”; 9288 bp) was a gift from Feng Zhang 16 (Addgene plasmid \#48138) (Fig. S3) [21], and SP-dCas9-VPR (“dCas9-VPR”; 11,319 bp) was a 17 gift from George Church (Addgene plasmid \#63798) (Fig. S4) [22]. sgRNA expression vector 18 (3028 bp) harboring a U6 promoter positioned upstream of the customized sgRNA sequence was 19 obtained from Genscript (USA) and confirmed by DNA sequencing. The sequences of sgRNA 20 are shown in Table S1. Fibroblast growth factor-2 was purchased from Peprotech (USA). M21 MLV Reverse Transcriptase was obtained from Promega (USA). Human GDNF Duoset ELISA 22 kit was purchased from R\&D system (USA). Label IT nucleic acid labeling kit was obtained 
1 from MirusBio (USA). SingleShot SYBR Green One-Step Kit and iTaq Universal SYBR Green

2 One-Step Kit were purchased from Bio-Rad (USA). hMSCs were purchased from Lonza

3 (Switzerland). All other cell culture reagents, Quant-iT PicoGreen dsDNA assay kit, Click-iT

4 EdU cell proliferation assay kit and SYBR Select Master Mix were purchased from

5 ThermoFisher (USA).

\section{2.2. Fabrication of LbL SAP-coated nanofibers}

8 Electrospun nanofibers were fabricated according to our previous report [18]. In brief, small 9 strips of PCL films with thickness of $20 \mu \mathrm{m}$ were adhered onto coverslips $(\varnothing 18 \mathrm{~mm})$ to create a $101 \mathrm{~cm}^{2}$ area, and the coverslips were then placed on a rotation wheel $(2400 \mathrm{rpm})$ for the collection 11 of electrospun nanofibers. The PCL solution (14 wt\% in TFE) was loaded into a syringe and 12 dispensed at a fixed rate $\left(1.0 \mathrm{~mL} \mathrm{~h}^{-1}\right)$ by a syringe pump. Voltages of $+8 \mathrm{kV}$ and $-4 \mathrm{kV}$ were 13 then applied to the blunt needle tip and the rotating collector, respectively. The distance between 14 spinneret and collector was set as $22 \mathrm{~cm}$. The obtained electrospun fibers were sterilized by UV 15 irradiation for $30 \mathrm{~min}$ followed by incubation in $70 \%$ ethanol for $10 \mathrm{~min}$.

$16 \mathrm{SAP}^{-}$and $\mathrm{SAP}^{+}$-RGD peptide powders were dissolved in sterilized $0.1 \mathrm{~mm} \mathrm{NaOH}$ solution $(1 \%$, $17 \mathrm{w} / \mathrm{v})$ and $0.1 \mathrm{~mm} \mathrm{HCl}(1 \%, \mathrm{w} / \mathrm{v})$, respectively, and sonicated for $30 \mathrm{~min}$ prior to use. To get the $18 \mathrm{LbL}$ assembled scaffolds, $50 \mu \mathrm{L}$ of $\mathrm{SAP}^{-}$solution $(0.2 \%, \mathrm{w} / \mathrm{v})$ was firstly dripped onto the PCL 19 nanofibers to cover the $1 \mathrm{~cm}^{2}$ scaffold area. After incubation at $37^{\circ} \mathrm{C}$ for $2 \mathrm{~h}$, the scaffolds were 20 rinsed with DI $\mathrm{H}_{2} \mathrm{O}$ to remove uncombined peptides. $4 \mu \mathrm{L}$ of PEIpro $(1 \mathrm{mg} / \mathrm{mL})$ were diluted in 21 DI $\mathrm{H}_{2} \mathrm{O}$ before complexation with $4 \mu \mathrm{g}$ of plasmids $(\mathrm{N} / \mathrm{P}=8)$. The complexation was performed 
1 at room temperature for $15 \mathrm{~min}$ and then placed onto the scaffolds to allow adsorption at $37{ }^{\circ} \mathrm{C}$

2 for $1 \mathrm{~h}$. The unbonded complexes were removed by washing with $\mathrm{DI} \mathrm{H}_{2} \mathrm{O}$, and $50 \mu \mathrm{L}$ of SAP S $^{+}$

3 RGD solution $(0.2 \%, \mathrm{w} / \mathrm{v})$ was then added onto the scaffolds. After incubation at $37^{\circ} \mathrm{C}$ for $2 \mathrm{~h}$,

4 the scaffolds were rinsed again with $\mathrm{DI} \mathrm{H}_{2} \mathrm{O}$ to remove uncombined peptides. To investigate the

5 sequential alternation of surface charges during LbL coating, the PCL nanoparticles $(\varnothing \sim 400 \mathrm{~nm})$

6 were coated with the same protocol and the zeta-potential was measured by Malvern Nano-ZS

7 Zeta Sizer after each step. The experiment was repeated 3 times.

9 2.3. SEM characterization of scaffolds

10 The lyophilized samples were sputter-coated with platinum at $10 \mathrm{~mA}$ for $100 \mathrm{~s}$, and then the 11 surface morphology of the scaffolds was observed under a scanning electron microscope (SEM, 12 JOEL, JSM-6390LA) at a 10-kV accelerating voltage. The average fiber diameters were 13 measured by ImageJ (NIH) with at least 100 fibers per sample.

\section{$15 \quad$ 2.4. Water contact angle measurements}

16 To study the surface wettability of SAP-coated PCL scaffolds, the static water contact angles of 17 PCL, PCL@SAP ${ }^{-}$and PCL@SAP $@ S^{-} \mathrm{SAP}^{+}-\mathrm{RGD}$ scaffolds were measured by the sessile drop 18 method using a contact angle analyzer (Kruss DSA 25). The droplet volume was set as $2 \mu \mathrm{L}$. 19 Measurements were carried out at least 3 times for each scaffold. 
2 To evaluate changes in chemical bond characteristics of the PCL scaffolds before and after SAP

3 coating, FTIR was carried out by using a Fourier-transform infrared spectrometer (PerkinElmer

4 Spectrum One). Spectra were acquired in the $400-4000 \mathrm{~cm}^{-1}$. The samples were subjected to 5 flash freezing and lyophilization prior to the measurement.

7 2.6. Characterizations of complex loading and release

8 To visualize the distribution of pDNA/PEIpro complexes on the scaffolds, pDNA was tagged 9 with MFP488 using Label IT nucleic acid labeling kit as per the manufacturer's instructions. The 10 MFP488-tagged pDNA was then complexed with PEIpro and adsorbed onto the scaffolds 11 according to the protocol mentioned above. The scaffolds were then visualized under an 12 epifluorescence microscope (Leica DMi8).

13 To test the loading efficiency of the pDNA/PEIpro complexes on the scaffolds, the rinsate 14 during coating processes was collected carefully. After de-complexion from the pDNA/PEIpro 15 complexes using heparin sodium salt $\left(1: 1 \mathrm{v} / \mathrm{v}, 20 \mu \mathrm{g} \mathrm{mL} \mathrm{m}^{-1}\right)$, the amount of pDNA in the rinsate 16 was determined using the Quant-iT PicoGreen dsDNA assay kit according to manufacturer's 17 protocol. The experimental loading efficiencies were computed using the following equation:

Loading efficiency $(\%)=\frac{m_{t}-m_{u}-m_{1}}{m_{t}} \times 100 \%$ 
1 where $m_{t}$ is the total mass of pDNA used for loading, $m_{u}$ is the mass of unbound pDNA (pDNA

2 remained in the complex coating solution) and $m_{1}$ the mass of pDNA loss during the coating of

$3 \mathrm{SAP}^{+}-\mathrm{RGD}$ (pDNA released into the $\mathrm{SAP}^{+}-\mathrm{RGD}$ coating solution).

4 Subsequently, the scaffolds were incubated with $1 \mathrm{~mL}$ of PBS at $37{ }^{\circ} \mathrm{C}$. While culture media

5 better mimics the cell culture condition, the other components in the culture media or serum

6 protein may affect the DNA assay reactions and plate reader readings. At each time point, $1 \mathrm{~mL}$

7 of PBS was collected from each sample and replaced with an equal volume of fresh PBS. The

8 supernatant was then used to measure the amount of pDNA by the same protocol mentioned 9 above.

\section{2.7. Cell culture}

12 U2OS cells were a generous gift from Professor Wenting Zhao at Nanyang Technological 13 University. The U2OS cells and hMSCs were cultured in high-glucose DMEM with GlutaMAX, 14 supplemented with $10 \%$ fetal bovine serum and $1 \%$ penicillin/streptomycin. The medium change 15 was performed every 2-3 days. hNPCs were generated from induced-pluripotent stem cells as 16 described previously [23]. The cells were cultured on poly-L-ornithine and laminin-coated 17 culture dishes in maintenance medium, which consisted of DMEM/F12 supplemented with $1 \%$ 18 glutaMAX, $1 \% \mathrm{~N} 2$ supplement, 2\% B27 supplement, $1 \%$ penicillin/streptomycin and $20 \mathrm{ng} \mathrm{mL}^{-1}$ 19 FGF2. All the cells were cultured in a humidified incubator at $37{ }^{\circ} \mathrm{C}$ and $5 \% \mathrm{CO}_{2}$. Passage 6 20 hMSCs and passage $9 \mathrm{hNPCs}$ were used in this study. 


\section{2.8. Cell adhesion, proliferation and scaffold-mediated local transfection}

2 To assess the effects of different surface coatings on cell adhesion, U2OS and hMSCs were

3 seeded at a density of 10,000 cells $\mathrm{cm}^{-2}$, while hNPCs were seeded at a density of 50,000 cells

$4 \mathrm{~cm}^{-2}$. To assess the effects of the SAP-coating on cell proliferation, the cell-seeded scaffolds

5 were incubated with $10 \mathrm{~mm}$ EdU for $24 \mathrm{~h}$ before being fixed with $4 \%$ PFA. The scaffolds were

6 permeabilized with $0.25 \%$ Triton-X then stained with the Click-iT reaction cocktail and Hoechst

7 33342. In each group, over 10 images from at least 3 scaffolds were taken at $20 \times$, and at least

8200 cells were quantified. The number of nuclei and EdU labelled nuclei were counted. For

9 scaffold-mediated local transfection experiments, the Cas9-GFP plasmid was used, in which the

10 2A-GFP was fused to Cas9 to allow the detection of Cas9 expression in the transfected cells, and

11 the U2OS cells were also seeded at a density of 10,000 cells cm$~_{-2}$. After incubation at $37{ }^{\circ} \mathrm{C}$ for

$1248 \mathrm{~h}$, the cells were fixed with $4 \%$ PFA solution for 10 min and then permeabilized in $0.25 \%$

13 Triton-X 100 in PBS for $15 \mathrm{~min}$ at room temperature. For morphology studies, the cytoskeleton 14 was stained with Alexa Fluor 555 phalloidin or Alexa Fluor 488 phalloidin, and nuclei were 15 stained with DAPI. Fluorescent images were acquired with an epifluorescence microscope (Leica 16 DMi8) and analyzed using Image $\mathrm{J}(\mathrm{NIH})$. In each group, over 10 images from at least 3 17 scaffolds were taken using a $20 \times$ objective, and at least 50 cells were quantified. The circularity 18 of cells was computed using the following equation:

20 where A is the area occupied by cell and P is the perimeter of cell. Higher circularity means less 21 spreading. In addition, the total cell number was counted by DAPI, while the $\mathrm{GFP}^{+}$cells were 22 counted based on the co-localization of GFP and DAPI signals. 
3 To assess the biocompatibility of the SAP-coated scaffolds in vivo, $1 \times 1 \mathrm{~cm}$ PCL fiber scaffolds 4 with thickness of $94 \pm 4.7 \mu \mathrm{m}$ were prepared with or without SAP coating according to the 5 procedure mentioned. All experimental procedures that involved animals were performed in 6 accordance with the Nanyang Technological University's Institutional Animal Care and Use 7 Committee, (IACUC, NTU) guidelines. Sprague-Dawley rats (8 weeks, 200-250g) were 8 anesthetized with isoflurane before shaving their backs. Thereafter, subcutaneous pockets were 9 created using surgical scissors after $1.2 \mathrm{~cm}$ incisions were made beside the spinal column. 10 Following that, the scaffolds were inserted into the subcutaneous pockets such that each animal 11 received both plain and SAP-coated scaffolds. The wounds were then closed with suture and the 12 animals were observed for 5 days. After 5 days, animals were sacrificed and the tissues enclosing 13 scaffolds were harvested and fixed in 4\% PFA overnight. Thereafter, the scaffolds were 14 dehydrated, embedded in paraffin and sectioned to obtain $5 \mu \mathrm{m}$ thick sections. The sections were 15 then stained with Haematoxylin and Eosin (H\&E) and imaged with ZEISS Axio Scan.Z1 using a $1620 \times$ objective. The images were then analyzed by an experimenter blinded to experimental 17 groups. Semi-quantitative evaluation was performed on the scaffold biocompatibility based on 18 fibrotic tissue formation, presence of macrophages (rounded morphology) at the boundary of the 19 scaffold, and fibroblasts (elongated morphology) infiltration into the scaffold; using a scoring 20 system, +: minimal, ++ : some, +++ : moderate, ++++ : significant $(\mathrm{n}=4$ rats $)$. To further evaluate 21 inflammatory response towards the scaffolds, the number of macrophages and neutrophils in the 22 scaffolds were counted. A total of 18 evenly distributed ROIs $(50 \mu \mathrm{m} \times 50 \mu \mathrm{m})$ were assessed 23 for each sample ( $n=4$ rats). 


\section{2.10. Bolus transfection experiments}

3 A preliminary test has been performed with different transfection reagents, including 4 Lipofectamine 3k, L-PEI, PEIpro, and JetOPTIMUS (Fig. S14). PEIpro demonstrated one of the 5 highest transfection efficiency and acceptable toxicity and hence was used for the subsequent 6 experiments. For gene activation experiments, U2OS cells were seeded onto 24-well plates at a 7 density of 20,000 cells per well. Twenty-four hours after plating, cells were transfected with 8 Cas9-GFP plasmids or a 1:1 mass ratio of dCas9 plasmid:sgRNA plasmids. A total plasmid mass 9 of $0.5 \mu \mathrm{g}$ per well was transfected using $0.5 \mu \mathrm{L}$ per well of PEIpro reagent. For synergy 10 experiments, the amount of total sgRNA was kept constant and the mass ratio of each sgRNA 11 was $1: 1$ or 1:1:1. The cells were harvested $2 \mathrm{~d}$ after transfection, and gene expression analysis 12 was performed.

14 2.11. Scaffold-mediated gene activation in mammalian cells

15 For exogenous gene-activation experiments, the dCas9 expression plasmids and sgRNA 16 plasmids were co-delivered with a mass ratio of $1: 1$, and the sub-mass ratio of each sgRNAs was 17 1:1:1. U2OS cells were seeded onto the scaffolds at a density of 10,000 cells $\mathrm{cm}^{-2}$ in $200 \mu \mathrm{L}$ 18 culture medium. The medium was sufficient to cover the $1 \mathrm{~cm}^{2}$ scaffold area. Three experiments 19 were performed. Gene activation was verified by gene expression analysis (Section 2.12). The 20 amount of GDNF secreted by the U2OS cells on the scaffolds was quantified by ELISA. 21 Specifically, the $200 \mu \mathrm{l}$ medium was collected every $24 \mathrm{~h}$ and replaced with $200 \mu \mathrm{L}$ fresh 
1 medium for 3 days, and the collected medium was then stored at $-80{ }^{\circ} \mathrm{C}$ for subsequent assays

2 (Section 2.13). To test the bioactivity of the secreted GDNF, medium was collected after 3 days

3 of culture without medium replacement. Thereafter, the collected medium was added to the

4 cortical neurons and DRG explant cultures as conditioned medium (Section 2.14).

$6 \quad$ 2.12. Gene expression analysis

7 After 2 days of culture, cells seeded on the 24-well plates were lysed by TRIzol reagent, and 8 RNA was extracted according to the manufacturer's instructions. The RNA concentration was 9 determined by the NsD-1000 spectrophotometer (NanoDrop Technologies), and 500 ng of RNA 10 from each sample were reverse transcribed into cDNA using M-MLV Reverse Transcriptase. 11 Real-time PCR was performed on the StepOnePlus system (Applied Biosystems) using SYBR 12 Select Master Mix. The sequences of the primers are listed in Table S2 and GAPDH was used as 13 the housekeeping gene. The primers were checked to have similar amplification efficiency. The 14 PCR parameter was 10 min at $95{ }^{\circ} \mathrm{C}$, followed by $15 \mathrm{~s}$ at $95^{\circ} \mathrm{C}$ and $1 \mathrm{~min}$ at $60{ }^{\circ} \mathrm{C}$ for 40 cycles. 15 The relative gene expression was calculated using the $\Delta \Delta \mathrm{C}_{\mathrm{T}}$ method, where the fold difference 16 was calculated using the expression $2^{-\Delta \Delta C_{T}}$. For cells that were seeded on the scaffolds, the cells 17 were lysed by SingleShot SYBR Green One-Step Kit and PCR was performed with iTaq 18 Universal SYBR Green One-Step Kit according to the manufacturer's instructions. 
1 The amount of secreted GDNF in the medium was quantified using the media obtained in

2 Section 2.11. Specifically, after the scaffolds were functionalized with SAP coatings and

3 plasmids, U2OS cells were seeded onto the scaffolds at a density of 10,000 cells $\mathrm{cm}^{-2}$ in $200 \mu 1$

4 medium. The medium was collected every $24 \mathrm{~h}$ and replaced with $200 \mu \mathrm{l}$ fresh medium for three

5 days. The collected medium was stored in $-80{ }^{\circ} \mathrm{C}$ freezer until quantification and was measured

6 by using a human GDNF ELISA kit following the manufacturer' $s$ instructions without dilution.

7 In brief, 96-well microplate was coated with $100 \mu \mathrm{L} /$ well of the Capture Antibody and incubated

8 overnight at room temperature, followed by washing with wash buffer for three times. Thereafter,

9 non-specific binding was blocked with Reagent Diluent at room temperature for $1 \mathrm{~h}$ prior to

10 wash for three times. The samples and standards were then added into the coated wells $(100 \mu \mathrm{L}$

11 each) and incubated for $2 \mathrm{~h}$ at room temperature. The antigen was then incubated with $100 \mu \mathrm{L}$ of

12 Detection Antibody for $2 \mathrm{~h}$, followed by the solution of Streptavidin-HRP A for $20 \mathrm{~min}$ in dark.

13 The addition of Substrate Solution $(100 \mu \mathrm{L}$ each) started the color reaction which was stopped 20

14 min later with $50 \mu \mathrm{L}$ of Stop Solution. The optical density of each well was immediately

15 measured at $450 \mathrm{~nm}$ with a reference wavelength at $570 \mathrm{~nm}$. GDNF concentrations were

16 determined from the regression line for the GDNF standard ranging from 31.3 to $500 \mathrm{pg} / \mathrm{mL}$.

17 Each sample represents one scaffold. Medium from three control and CRISPRa-GDNF scaffolds 18 was measured.

20 2.14. Cortical neurons and DRG explants culture

21 To test for the bioactivity of the GDNF secreted by the U2OS cells on the functionalized 22 scaffolds, we transferred the conditioned medium to the neuron cultures and evaluated neurite 
1 outgrowth. The conditioned media was obtained by collecting the $200 \mu 1$ culture media from

2 U2OS cells cultured on the control or CRISPRa-GDNF scaffolds for 3 days as mentioned in

3 Section 2.11. Cortical neurons were isolated from P1 Sprague-Dawley rat pup brain cortices by

4 trypsin digestion at $37{ }^{\circ} \mathrm{C}$ for $15 \mathrm{~min}$. After the suspension was passed through a $70 \mu \mathrm{m}$ cell

5 strainer, the dissociated cortical neurons were seeded on aligned nanofiber scaffolds at 30,000

6 cells $\mathrm{cm}^{-2}$ in the conditioned medium at $37{ }^{\circ} \mathrm{C}$ [24]. DRG explants were harvested from P3 rat

7 pups and all the meninges from the DRGs were taken off. Two DRG explants were seeded onto

8 each aligned nanofiber scaffolds with $1 \mathrm{~cm}^{2}$ area. The cortical neurons and DRG explants were

9 then cultured in the $200 \mu \mathrm{l}$ of the collected conditioned medium (Section 2.11) at $37{ }^{\circ} \mathrm{C}$. After

10 cultured for 3 days, cortical neurons and DRG explants were fixed with 4\% PFA at room 11 temperature for $20 \mathrm{~min}$. After washing in PBS for 3 times, cortical neurons and DRG explants 12 were permeabilized with $0.3 \%$ Triton X-100 followed by blocking with $10 \%$ goat serum for $1 \mathrm{~h}$ 13 at room temperature. Thereafter, the samples were incubated with mouse anti- $\beta$ III-Tubulin (Tuj$141,1: 1000$ dilution) overnight at $4{ }^{\circ} \mathrm{C}$, followed by incubation in goat anti-mouse Alexa Fluor 555

15 secondary antibody for $2 \mathrm{~h}$ at room temperature. Cell nuclei were counterstained with DAPI. 16 Fluorescent images were acquired with an epifluorescence microscope (Leica DMi8) and 17 analyzed using Image $\mathrm{J}(\mathrm{NIH})$. At least 5 scaffolds of each group were used in this experiment. 18 For cortical neurons, 10 images were taken using a $20 \times$ objective for each scaffold and at least 1990 cells were quantified for each group. For each DRG, the ten longest neurites were measured 20 and averaged to determine the average neurite outgrowth length for each sample. At least 5 DRG 21 explants were analyzed from each group.

\section{2.15. Statistical analysis}


1 All values were presented as mean \pm standard deviation (S.D). Outlier analysis was performed to

2 exclude significant outliers from the subsequent analyses. One-way ANOVA and Tukey's post-

3 hoc tests were used for samples that passed the homogeneity test. Otherwise, Kruskal-Wallis and

4 Mann - Whitney U test were used for pairwise analysis. Student's t-test was used to compare 2

5 independent samples. Tests were conducted with a $95 \%$ confidence interval $(\alpha=0.05)$.

7 3. Results

\subsection{Preparation and characterization of LbL coated PCL nanofibers}

9 The self-assembling peptides ( $\left.\mathrm{SAP}^{-}, \mathrm{SAP}^{+}-\mathrm{RGD}\right)$ were synthesized by Fmoc solid-phase 10 method. High-pressure liquid chromatography (HPLC) confirmed that the purity of the peptides 11 was $97.8 \%$ and $99.2 \%$, respectively, and the mass spectrometry results assured the correct 12 molecular weight of these peptides (Fig. S1, S2). The PCL nanofibers were fabricated using an 13 electrospinning technique, and the LbL coating was then performed based on the strategy as 14 illustrated in Scheme 1. SAP', the amphiphilic peptide bearing negative charges, was firstly 15 coated onto the PCL scaffolds by dip-coating, resulting in the negative surface charge. 16 Subsequently, the positively charged pDNA/PEIpro complexes and SAP ${ }^{+}$RGD were coated. 17 This assembly process can also take place on the surface of PCL nanoparticles and was 18 confirmed by the sequential alternation of zeta-potential (Fig. S5). The strong hydrophobic 19 interactions between the aromatic side chains of the $\beta$-sheet $\mathrm{SAP}^{-}$and the PCL molecules 20 allowed the adsorption of $\mathrm{SAP}^{-}$onto the hydrophobic PCL surface, while the established 21 negatively charged surface further facilitated the adsorption of pDNA/PEIpro complexes and 22 subsequently $\mathrm{SAP}^{+}$-RGD through electrostatic interactions. 

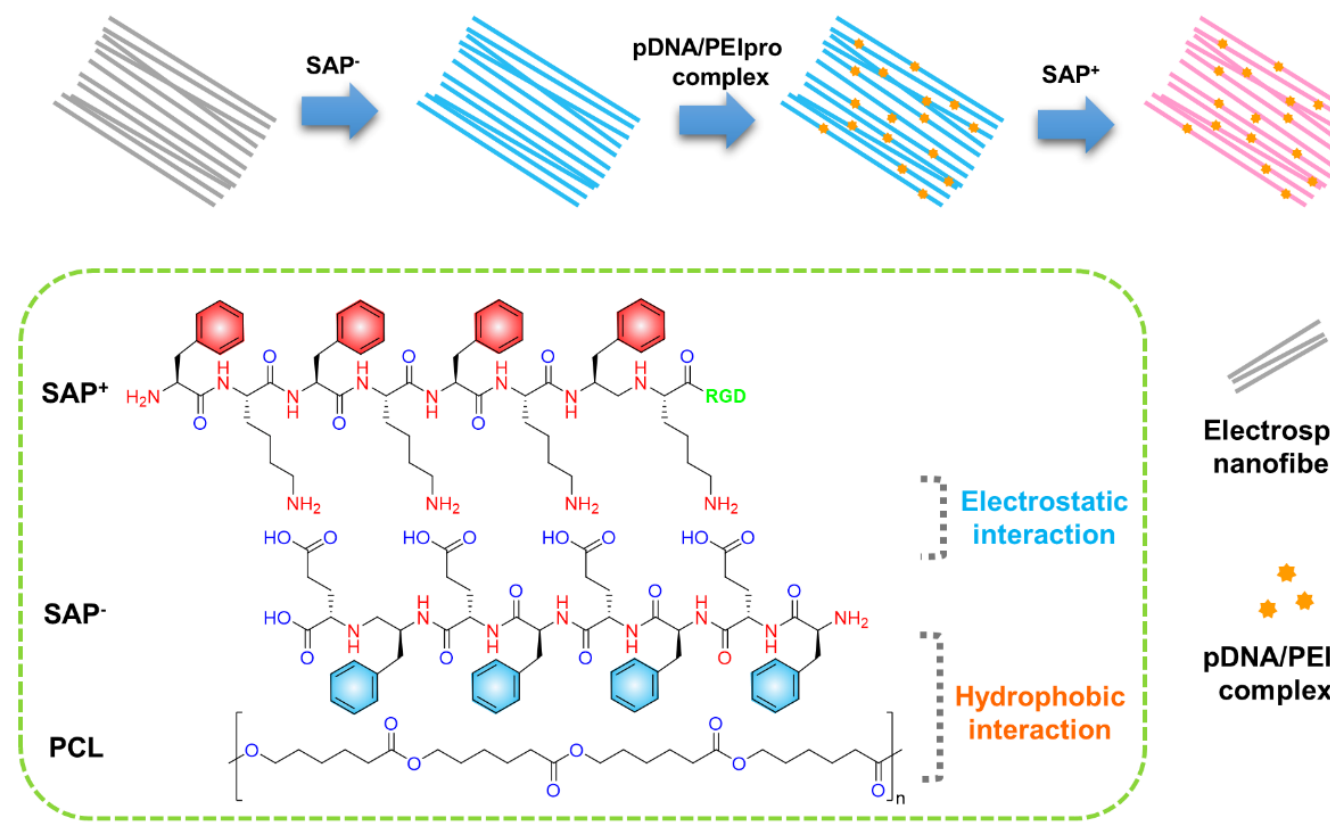

Electrospun

nanofibers

2 Scheme 1. Schematic illustration of the layer-by-layer coating of SAPs on PCL nanofibers.

3 As shown in the SEM images of the scaffolds (Fig. 1a), the SAP coatings did not mask the

4 underlying fiber morphology. Specifically, the average fiber diameter of the plain PCL scaffolds

5 was $929 \pm 107 \mathrm{~nm}$, while that of the $\mathrm{SAP}^{-}$-coated scaffolds ("PCL@SAP")) and SAP/SAP

6 RGD dual-coated scaffolds (“PCL@SAP'@SAP+-RGD”) was $945 \pm 103$ nm and $958 \pm 96$ nm,

7 respectively. This suggests that the SAP coatings did not significantly alter the topography of the

8 aligned fibers.

9 Surface wettability is one of the most important surface properties that affect the cellular 10 response towards an implanted substrate, including cell attachment, spreading, and proliferation. 11 The contact angles of PCL and PCL@ $\mathrm{SAP}^{-}$were $99.3 \pm 3.0^{\circ}$ and $35.3 \pm 2.9^{\circ}$, respectively. The 12 significantly increased hydrophilicity suggests the successful coating of the negatively charged 13 SAP $^{-}$. Subsequently, the adsorption of the positively charged PEI complexes and then the coating 14 of $\mathrm{SAP}^{+}-\mathrm{RGD}$ resulted in a slightly increased contact angle $\left(52.3 \pm 5.2^{\circ}\right)$. The contact angles of 
1 the SAP-coated PCL scaffolds did not change significantly after incubation in phosphate-

2 buffered saline (PBS) for at least 7 days (Fig. S6), indicating the good stability of SAP

3 modification. Furthermore, we also characterized these scaffolds by comparing typical chemical

4 bonds before and after SAP coating. Fourier transform infrared (FT-IR) spectroscopy

5 measurements revealed plain PCL scaffolds exhibited the absorption peak at $1720 \mathrm{~cm}^{-1}$, which is

6 indicative of the $-\mathrm{C}=\mathrm{O}$ stretching vibration of PCL (Fig. S7). After $\mathrm{SAP}^{-}$coating, two strong

7 peaks at $3293 \mathrm{~cm}^{-1}$ and $1635 \mathrm{~cm}^{-1}$ were observed, and these were attributed to the -NH-

8 stretching vibration and $-\mathrm{NH}_{2}$ bending vibration of $\mathrm{SAP}^{-}$, respectively. These findings

9 collectively suggest that the SAP was successfully coated onto the PCL nanofibers.

a
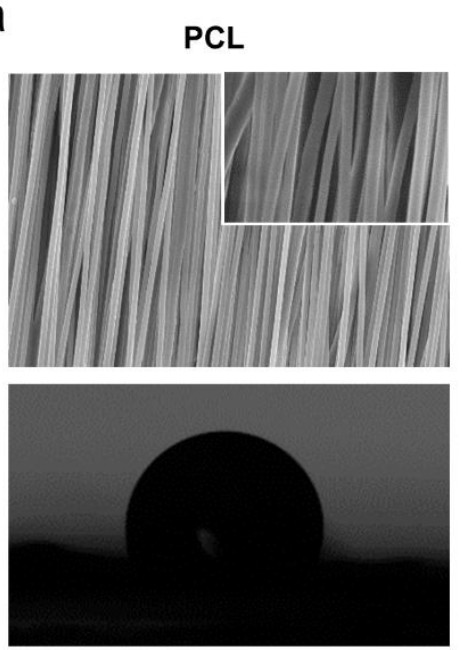

b

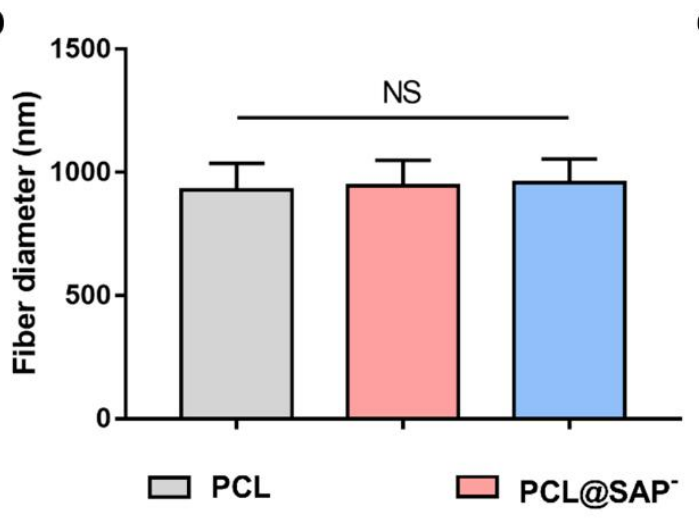

PCL@SAP-
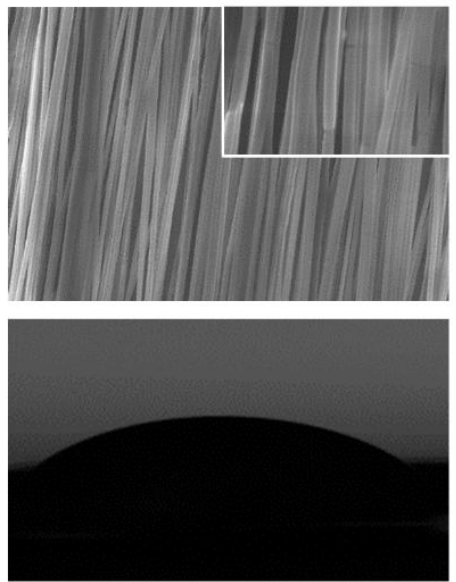

C

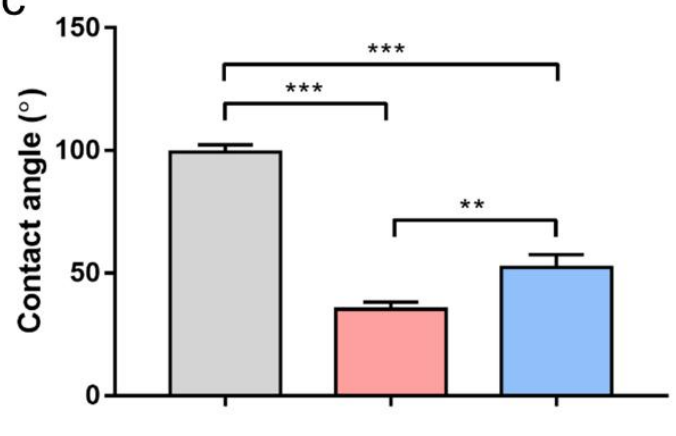

PCL@SAP'@SAP ${ }^{+}-R G D$

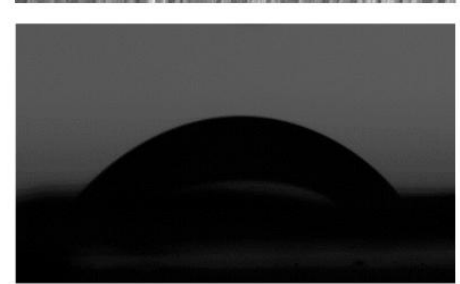

PCL@SAP-@SAP+-RGD

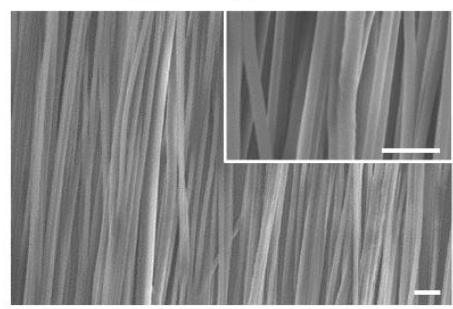


2 contact angles of the SAP-coated scaffolds; scale bar $=10 \mu \mathrm{m}$. b) Fiber diameters $(\mathrm{n}=3)$ and $\mathrm{c})$

3 water contact angles of the SAP-coated scaffolds $(\mathrm{n}=3) ;{ }^{* * *} p<0.001$, "NS" indicates "no 4 significance".

\subsection{SAP-coated scaffolds mediated efficient loading and sustained release of pDNA/PEIpro}

7 complexes

8 Polyethylenimine (PEI), a cationic polymer, is a widely used and cost-effective reagent for 9 gene delivery. However, the N-propionyl groups in PEI molecules may reduce the number of 10 protonatable nitrogen residues and hinder the nucleic acid condensation and endosomal escape. 11 Therefore, the deacylated PEI, commercially known as PEIpro, was chosen for this work and 12 shown to be superior for the mammalian cells [25]. As shown in Fig. S8, no significant 13 difference was observed in the particle size and zeta potential of PEIpro/pDNA complexes with 14 an increase in N/P ratio. Thus, the complexes of N/P $=8$ with moderate positive charge $(+8.4 \pm$ $15 \quad 1.1 \mathrm{mV})$ and relatively small size $(37.78 \pm 11.06 \mathrm{~nm})$ were chosen for all subsequent 16 experiments. To visualize the distribution of the pDNA/PEIpro complexes on the scaffolds, 17 fluorescently-tagged pDNA, MFP488-pDNA, was used. As shown in Fig. 2a, a uniform 18 distribution of MFP488-pDNA/PEIpro complexes could be identified on the aligned fiber 19 substrates. Specifically, the SAP-coated fibers, both PCL@SAP ${ }^{-}$and PCL@SAP $@ \mathrm{SAP}^{+}-\mathrm{RGD}^{-}$ 20 demonstrated significantly higher affinity to the complexes, and the loading efficiency of plain 21 PCL, PCL@SAP , and PCL@SAP @ SAP $^{+}$-RGD scaffolds was $82.9 \pm 2.4 \%, 97.2 \pm 2.6 \%$, and $2293.4 \pm 3.7 \%$, respectively (Fig. 2b). We next investigated the release kinetics of the complexes 
1 from the scaffolds that were incubated in PBS at $37{ }^{\circ} \mathrm{C}$ (Fig. 2c). As compared to the plain PCL

2 scaffolds in which the pDNA/PEIpro complexes were loosely entrapped, the SAP-coated

3 scaffolds exhibited significantly smaller initial burst release and slower subsequent release of the

4 complexes. These SAP-coated scaffolds were capable of releasing pDNA/PEIpro continuously

5 for at least a week. Notably, the PCL@SAP @SAP ${ }^{+}$-RGD scaffolds demonstrated similar

6 loading efficiency and release rate as that of the PCL@SAP${ }^{-}$scaffolds, indicating that the

7 subsequent coating of $\mathrm{SAP}^{+}$-RGD did not significantly affect the loading and release profile of

8 the complexes. It is believed that the electrostatic interactions between the negatively charged

$9 \mathrm{SAP}^{-}$and positively charged pDNA/PEIpro complexes may help to immobilize these complexes

10 on the scaffolds and slow down the release. In contrast, the faster release of complexes from the

11 plain PCL scaffolds may be due to the lack of such stabilizing mechanisms. Moreover, this

12 platform constructed by electrostatic interactions is also capable of trapping other complexes,

13 which include some of the commonly used RNA/Lipofectamine, protein/Lipofectamine, and $14 \mathrm{pDNA} / \mathrm{semiconducting} \mathrm{polymer} \mathrm{nanoparticles} \mathrm{(SPNs)} \mathrm{(Fig.} \mathrm{S9).} \mathrm{Hence,} \mathrm{the} \mathrm{platform} \mathrm{is} \mathrm{believed}$

15 to be an effective approach for the delivery of various positively charged nano-complexes. 
a
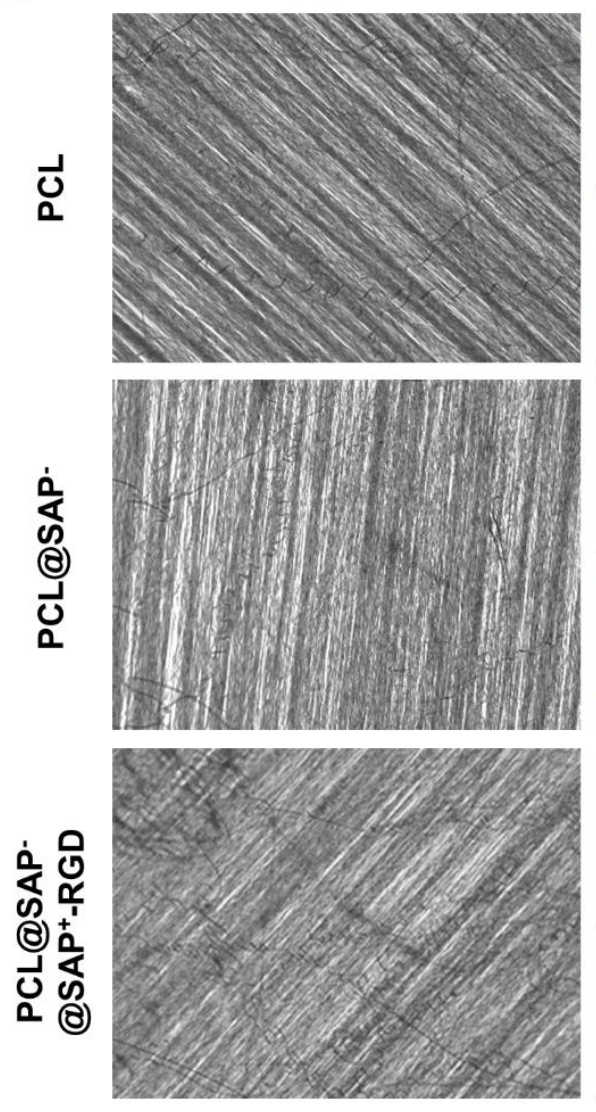

b

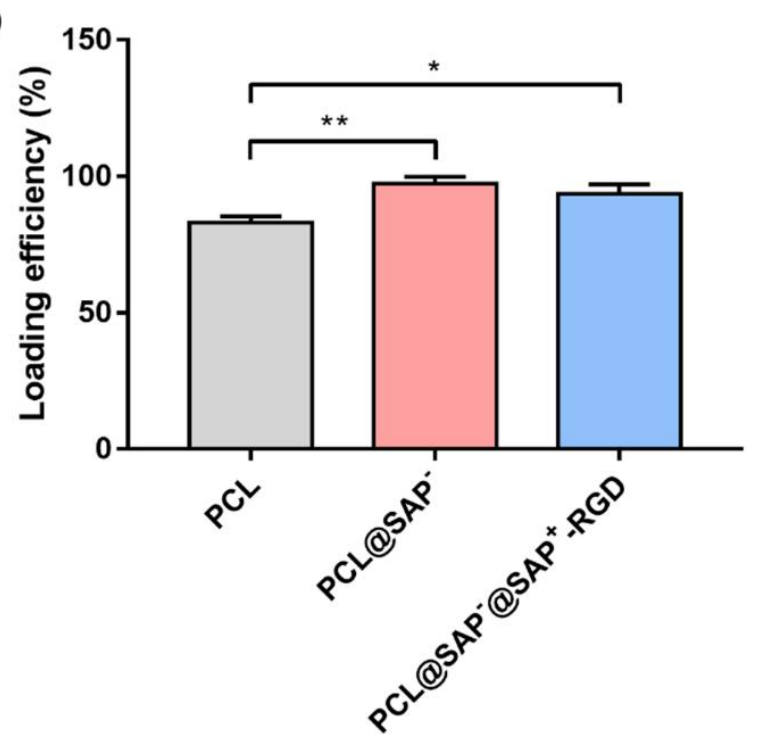

MFP488-pDNA
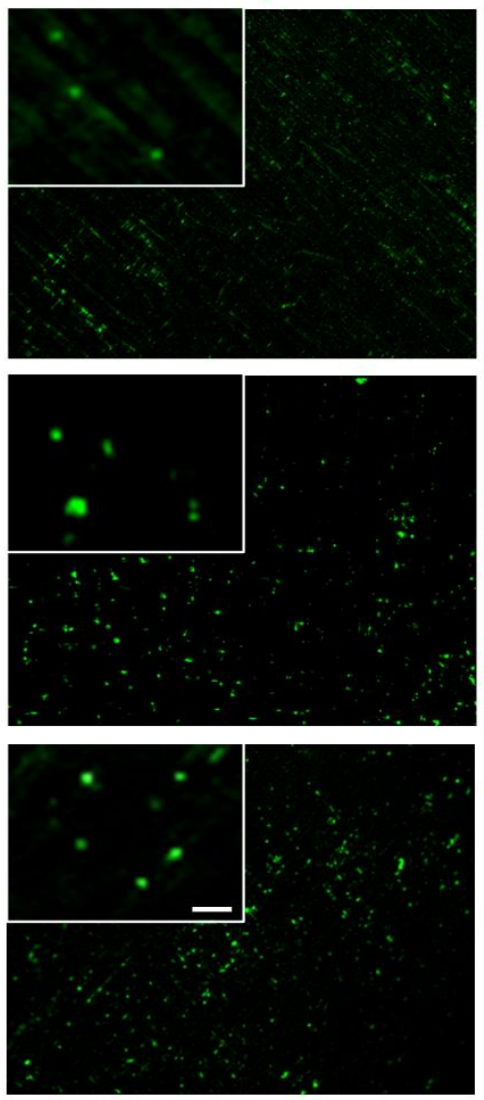

C

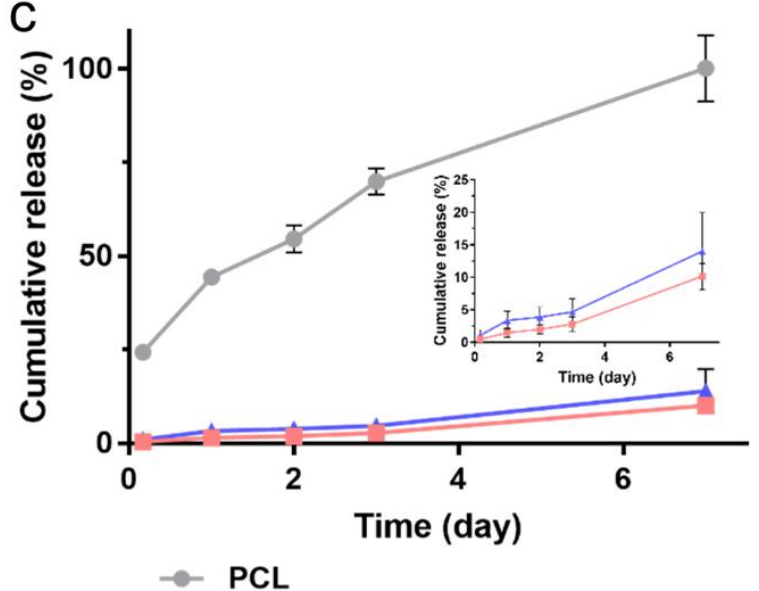

$\rightarrow$ PCL

-1-PCL@SAP

\ PCL@SAP'@SAP+-RGD
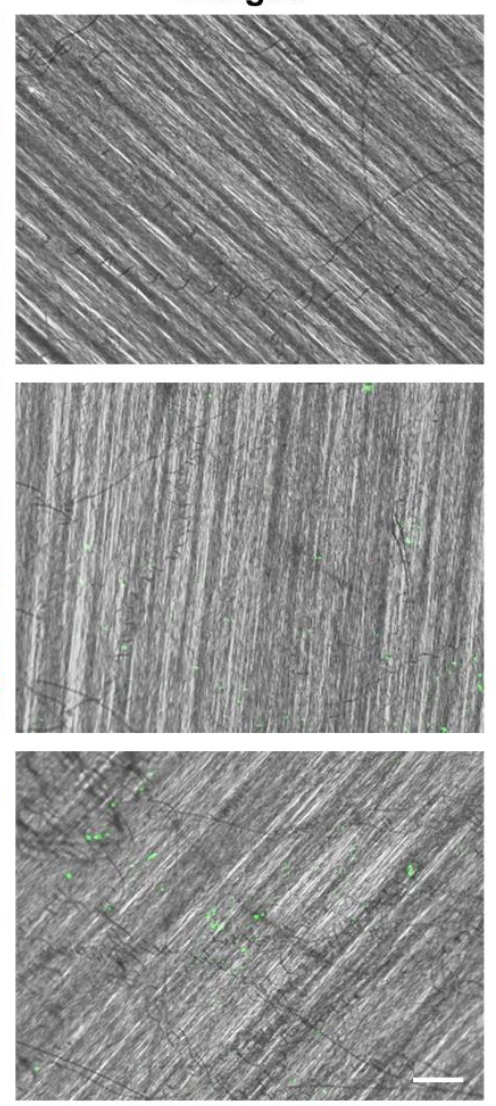
1 aligned fiber substrates. Scale bar $=100 \mu \mathrm{m}$ (Insert: $10 \mu \mathrm{m}$ ). b) Loading efficiency of

2 pDNA/PEIpro complexes on the scaffolds $(n=3)$. c) Cumulative release profile of

3 pDNA/PEIpro complexes from scaffolds over 7 days $(n=5)$.

4

$5 \quad 3.3$. SAP-coated scaffolds promoted cell adhesion and proliferation

6 To better visualize the nanofibers and cell-substrate interactions, Rhodamine B was linked to

7 the N-terminus of SAP' ${ }^{-}$We subsequently examined the effect of SAP coating on cell adhesion

8 by seeding U2OS cells on the scaffolds. As shown in Fig. 3 a, after 48 h of culture, only a limited

9 number of cells were observed on the plain PCL scaffolds, while significantly more cells were

10 observed on the PCL@SAP ${ }^{-}$and PCL@SAP @ SAP ${ }^{+}$-RGD scaffolds (Fig. 3b), indicating the

11 improved cell affinity. Similarly, when the cells were cultured in the serum-free medium, the

12 RGD-bearing scaffolds still demonstrated the best cell adhesion performance (Fig. 3c),

13 suggesting that the cells bound directly to RGD instead of the serum proteins that were present in

14 the culture medium. Other than U2OS, these SAP-coated scaffolds can also support the adhesion

15 and spreading of numerous cells, such as human mesenchymal stem cells (hMSCs) and human

16 neural progenitor cells (hNPCs) (Fig. S10). Besides, enhanced cell proliferation was also 17 observed on the PCL@SAP @SAP ${ }^{+}$-RGD scaffolds (Fig. S11). When these SAP-coated 18 scaffolds were subcutaneously implanted, they showed better biocompatibility and cell 19 infiltration in vivo (Fig. S12). 
a
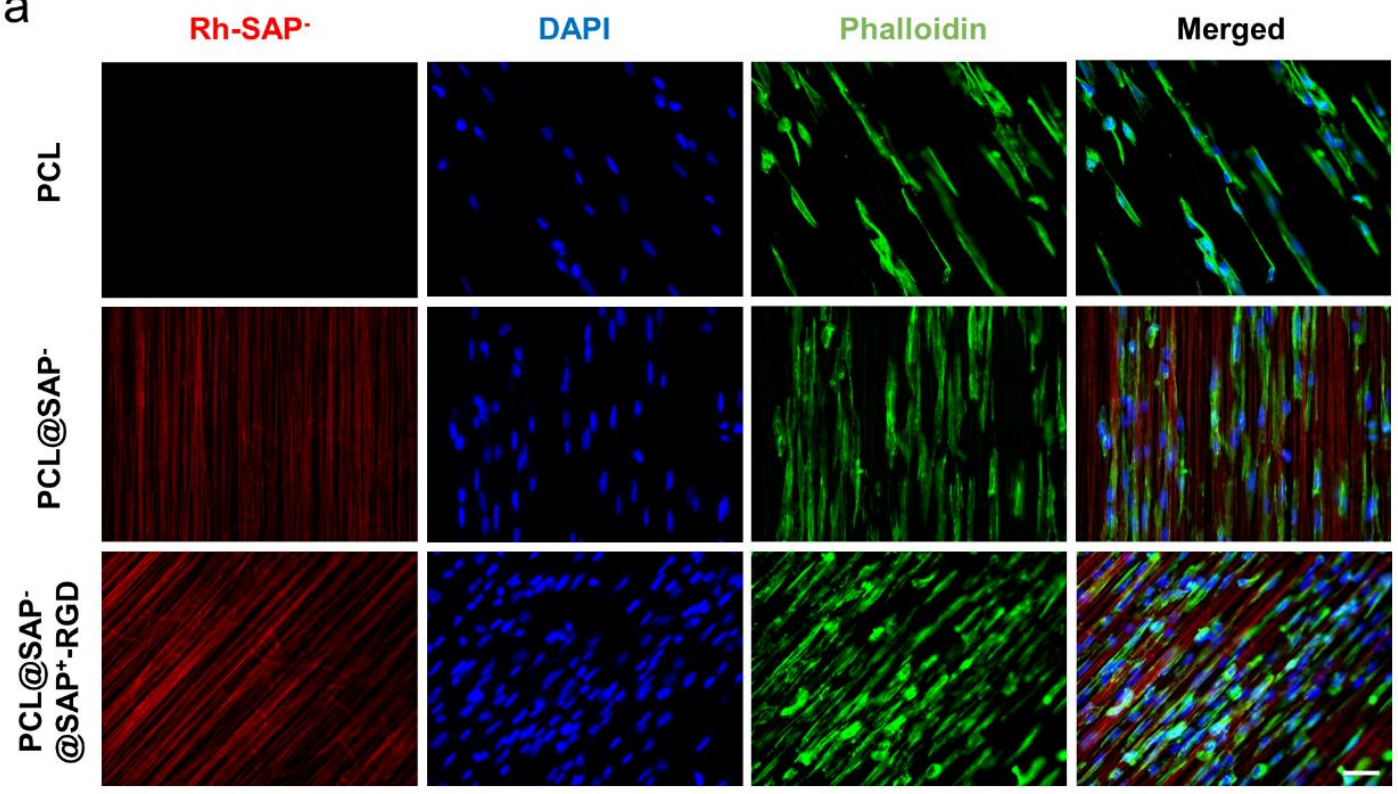

b

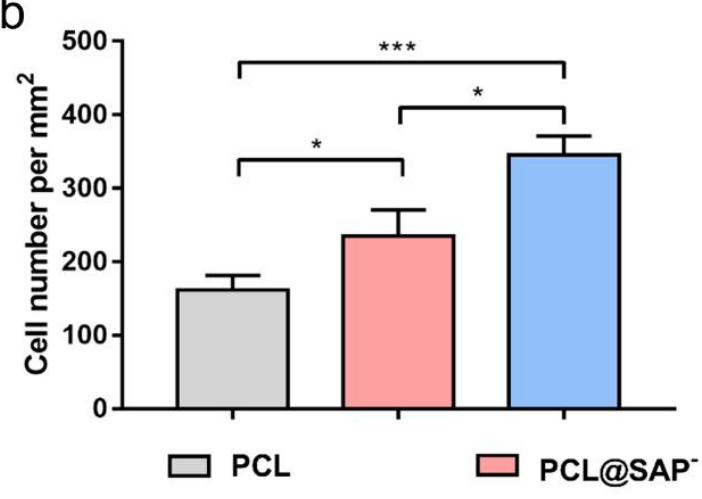

C

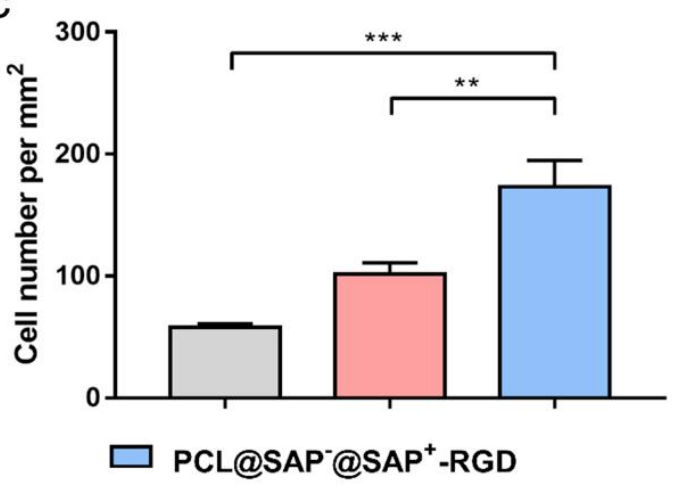


To facilitate the characterization of positive transfection using the CRISPR system, the

2 reporter gene, GFP, was fused to the Cas9 expression cassette [21], and the Cas9-GFP plasmids

3 were used for transfection. As shown in Fig. 4a, the PCL@SAP @SAP ${ }^{+}$-RGD group

4 demonstrated the greatest number of GFP-positive cells and the highest intensity of green

5 fluorescence. In comparison, the PCL@SAP ${ }^{-}$group showed a smaller number of GFP-positive

6 cells, and the green fluorescence was seldom observed in the absence of SAP coating ("PCL").

7 The quantitative results confirmed that PCL@SAP @ $\mathrm{SAP}^{+}-\mathrm{RGD}$ yielded $54.6 \mathrm{GFP}^{+}$cells per

$8 \mathrm{~mm}^{2}$, whereas PCL@SAP ${ }^{-}$and plain PCL only led to 14.4 and $0.7 \mathrm{GFP}^{+}$cells per $\mathrm{mm}^{2}$,

9 respectively (Fig. 4b). Meanwhile, although the total cell number on the PCL@SAP @ $\mathrm{SAP}^{+}$-

10 RGD scaffold was significantly higher than the others owing to the enhanced adhesion, this

11 group still showed the best $\mathrm{GFP}^{+}$percentage (16.1\%) among the three groups (Fig. 4c), which is

12 similar to the efficiency of the bolus transfection (18.2\%). Furthermore, the gene delivery

13 efficiency was dose-dependent (Fig. S13). Specifically, we found that $4 \mu \mathrm{g}$ was the optimal

14 amount of plasmid as we started to see toxicity and lower efficiency at $6 \mu \mathrm{g}$. We also attempted

15 to assess the transfection efficiency of hNPCs, which are more difficult to be transfected, by

16 seeding these cells on the scaffolds. Correspondingly, we were only able to achieve a much

17 lower transfection efficiency of $9.3 \%$ by scaffold-mediated delivery and $11.3 \%$ by bolus delivery 18 (Fig. S14). 
a

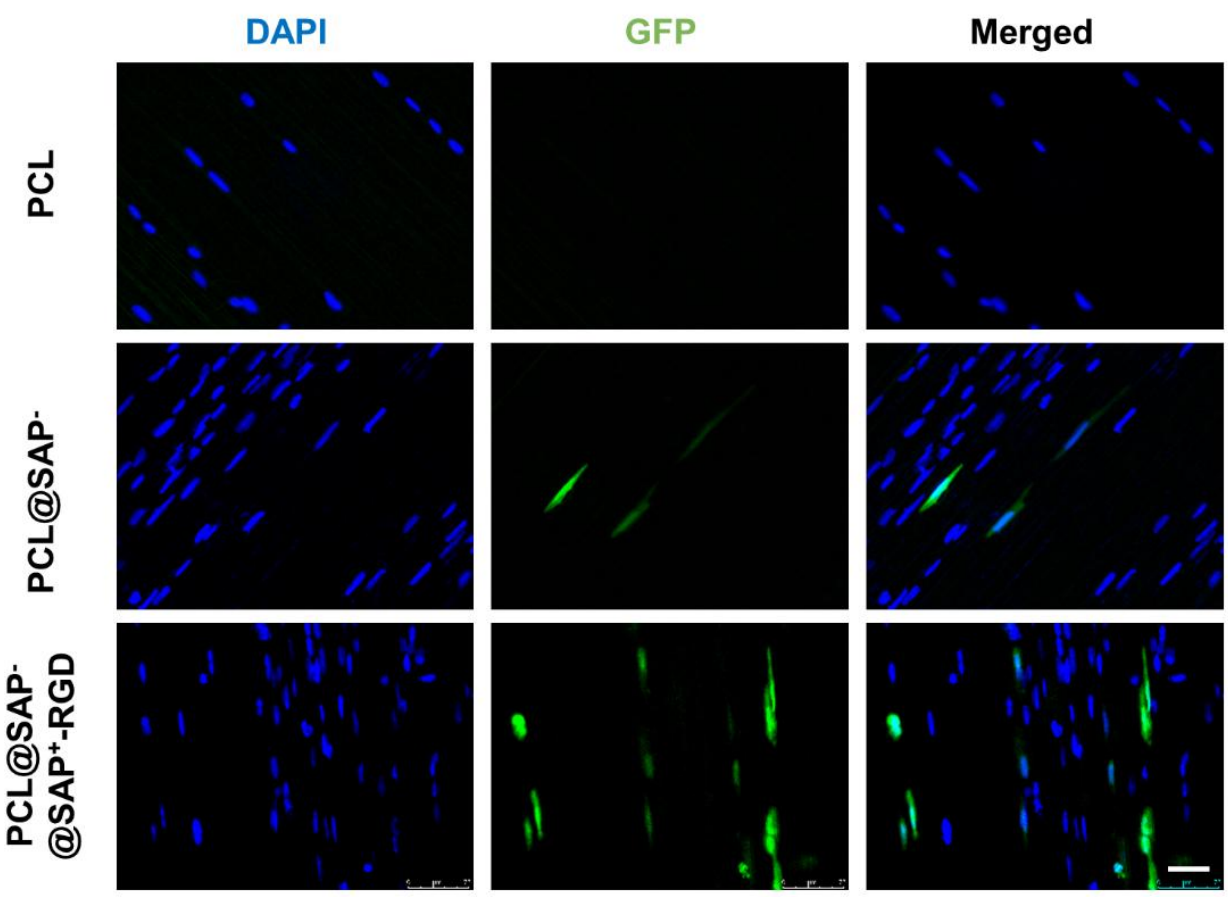

b

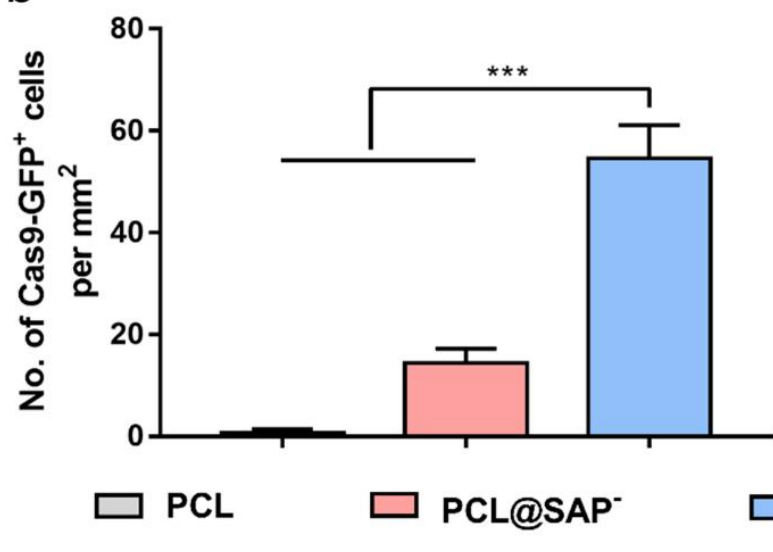

C

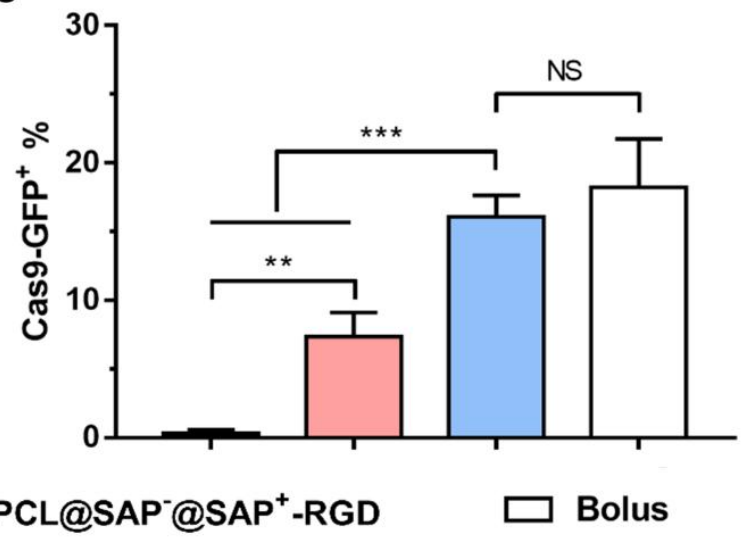

2 Fig. 4. SAP-coated scaffolds enhanced the efficiency of Cas9-GFP transfection in

3 mammalian cells. a) Representative fluorescent images of U2OS cells on different scaffolds;

4 scale bar $=50 \mu \mathrm{m}$. Quantification of b) average number of Cas9-GFP ${ }^{+}$cells per $\mathrm{mm}^{2}$ and c)

5 transfection efficiency based on fluorescent images $(\mathrm{n}=3) ;{ }^{* *} p<0.01,{ }^{* * *} p<0.001$, "NS"

6 indicates "no significance". 


\subsection{Scaffold-mediated CRISPRa system promoted endogenous gene-activation in mammalian}

2 cells

3 Having established the ability of the SAP-coated scaffolds to facilitate localized gene

4 transfection of mammalian cells, we next examined their specific performance in the delivery of

5 the CRISPRa system and constructed a CRISPRa system targeting hGDNF gene (Fig. 5a). Here,

6 we aimed to utilize GDNF as a proof of concept because the protein secreted is easily detected

7 and quantified, making it a straightforward evaluation of CRISPRa system delivery. The

8 sequences of $\operatorname{sgRNAs}$ reported in the literature were ligated into sgRNA expressing vectors and

9 confirmed by DNA sequencing (Table S1, Fig. S15) [26]. We first evaluated the sgRNAs in

10 U2OS cells via co-delivery of sgRNA and dCas9-VPR (i.e., dCas9 fused with a potent tripartite

11 activator, VP64, p65AD and Rta [22]) expressing vectors by bolus transfection, and the

12 treatment significantly increased the mRNA expression of GDNF (Fig. 5b). Meanwhile, it has

13 been reported that multiple sgRNA-dCas9-VP64 complexes can bind to a single promoter,

14 thereby acting synergistically and more efficiently [27]. This synergistic effect was also observed

15 in our experiments and hence the cocktail of sgRNAs $(\operatorname{sg} 01+02+03)$ was used in the 16 experiments hereafter.

17 Subsequently, these pDNA/PEIpro complexes of both sgRNAs and dCas9-VPR expressing 18 vectors were loaded onto the SAP-coated scaffolds, and the U2OS cells were seeded and 19 cultured on the scaffolds. Compared to the non-treated control group, scaffold-mediated 20 transfection of CRISPRa-GDNF resulted in substantial upregulation of GDNF mRNA expression 21 (Fig. 5c). In order to quantify the secretion of GDNF, ELISA was used for the assay of cell 22 culture medium at preset time points. Although the concentration of GDNF in the medium did 23 not change significantly after $24 \mathrm{~h}$, the cells started to produce robust levels of GDNF after $48 \mathrm{~h}$ 
1 (Fig. 5d), and the cumulative amount of GDNF secreted in the CRISPRa-GDNF group was also

2 higher than that in the control group (Fig. 5e). However, due to the fast proliferation rate, the

3 cells covered near $90 \%$ area of the scaffolds after $72 \mathrm{~h}$, and hence the monitoring of GDNF

4 expression had to be terminated. These results indicated that the CRISPRa system could be 5 loaded onto the scaffolds and delivered to recipient cells for gene engineering.

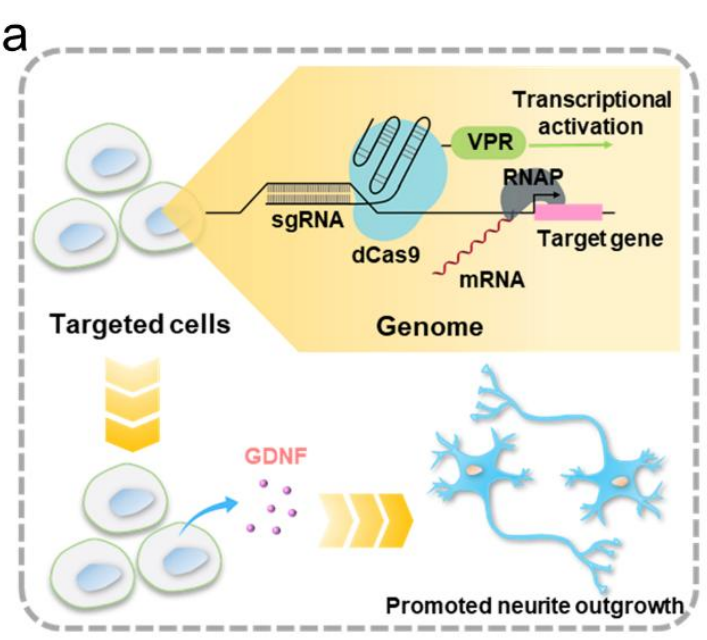

b

C

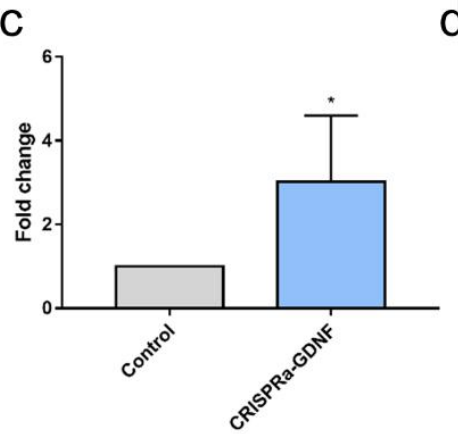

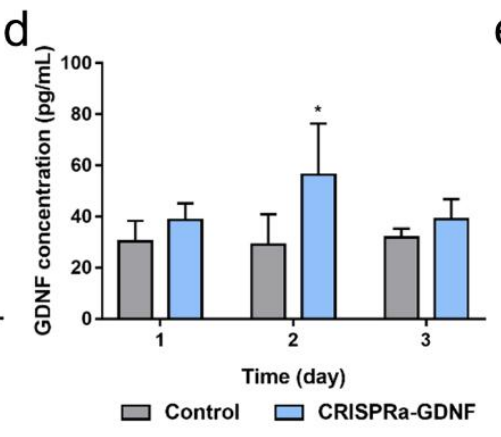

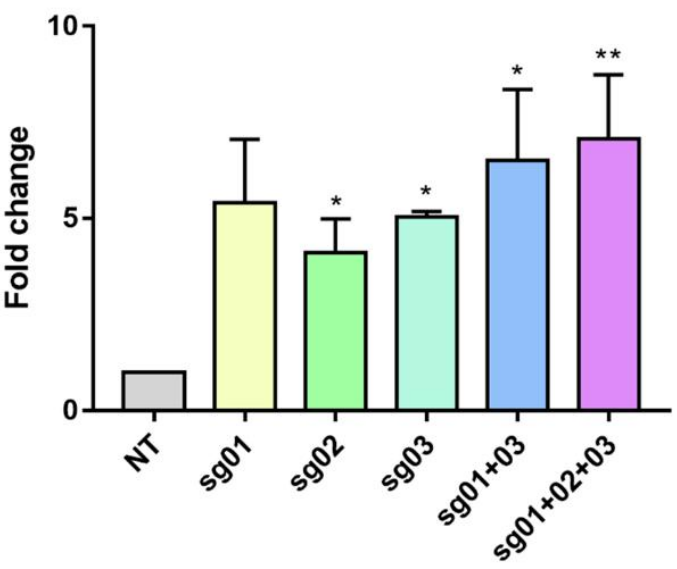

e

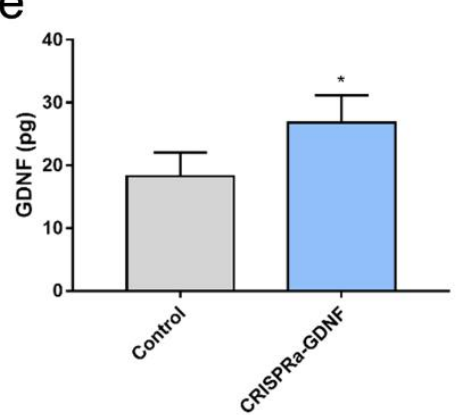

7 Fig. 5. SAP-coated scaffolds mediated the delivery of CRISPR/dCas9 based gene activation

(CRISPRa-GDNF) system to enhance GDNF mRNA and protein expressions. a) Schematic

9 representation of the CRISPRa-GDNF system. b) Relative expression of GDNF mRNA in U2OS

10 cells in which the individual or combinations of $\operatorname{sgRNAs}$ were co-delivered with dCas9-VPR (n $11=3$ ). c) Relative expression of GDNF mRNA, d) GDNF concentration in culture medium at 
1 different time points and e) cumulative GDNF protein expression over 3 days in U2OS cells

2 seeded onto the PCL@SAP $@ \mathrm{SAP}^{+}-\mathrm{RGD}$ scaffolds $(\mathrm{n}=3) ;{ }^{*} p<0.05,{ }^{* *} p<0.01$.

3 3.6. Scaffold-mediated CRISPRa-GDNF system promoted neurite outgrowth

4 To further investigate the functional effect of the scaffold-mediated CRISPRa-GDNF

5 system, the ideal experiment would have been a co-culture experiment of U2OS cells and

6 neurons. However, the complexity of keeping both cells alive in the same medium was difficult.

7 The U2OS cells had a high proliferative rate on the scaffolds and would compete for nutrients

8 with the neurons, without adding more media and supplements that might mask the effect of the

9 secreted GDNF. Therefore, we collected the media containing GDNF that were secreted by the

10 U2OS cells seeded on the CRISPRa-functionalized scaffold after 3 days (i.e. conditioned 11 medium), and tested for the bioactivity of the secreted GDNF using primary neurons. 12 Specifically, we transferred the conditioned medium to rat cortical neurons and dorsal root 13 ganglia (DRGs) explants that were cultured on the nanofiber scaffolds for another 3 days. Fig. 6a 14 and $\mathrm{b}$ show the representative fluorescent images of cortical neurons and DRGs cultured in 15 culture medium from control and CRISPRa-GDNF scaffolds, respectively, and the neurite 16 outgrowth along the long axis of fibers could be observed. Quantitative analysis confirmed that 17 the CRISPRa-GDNF conditioned medium significantly enhanced neurite outgrowth in both 18 cortical neurons and DRG explants (Fig. 6c and d). 
a
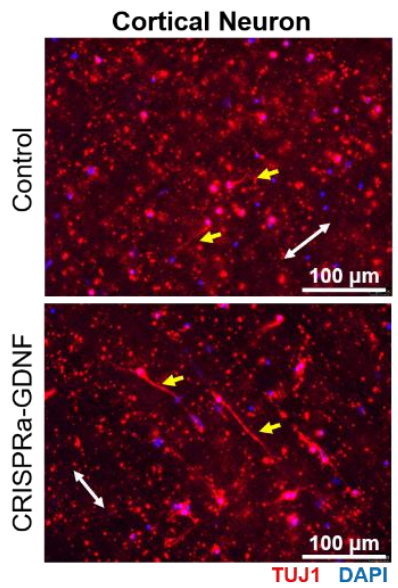

C

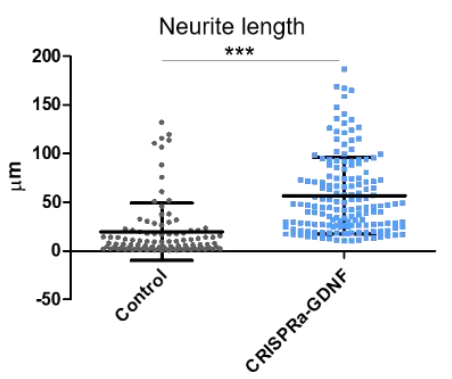

b
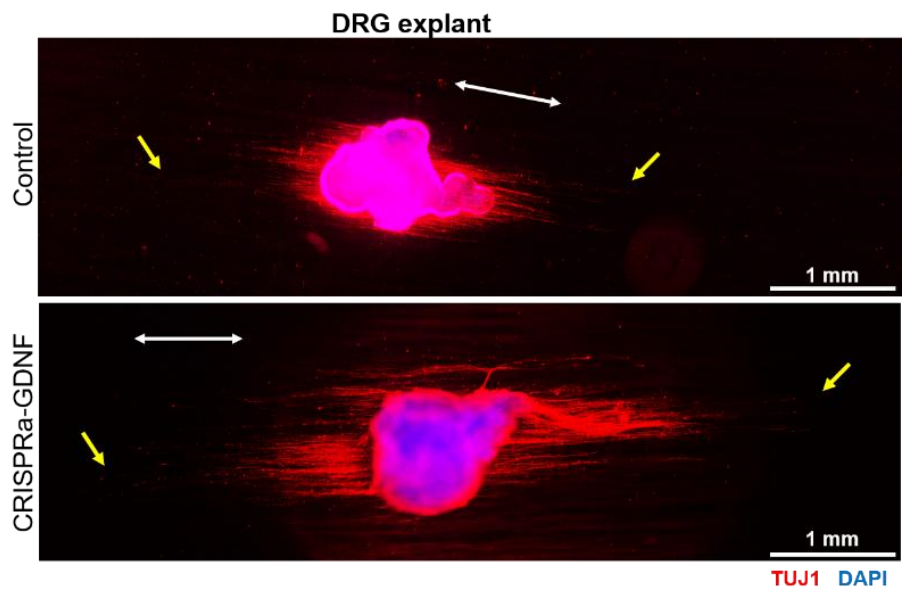

d

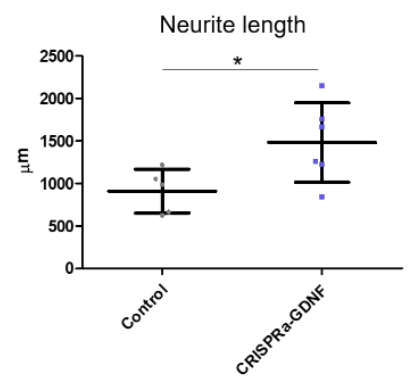

2 Fig. 6. Scaffold-mediated CRISPRa-GDNF system promoted neurite outgrowth.

3 Representative fluorescent images of a) rat cortical neurons and b) DRG explants cultured in

4 control culture medium and CRISPRa-GDNF conditioned medium, respectively. White arrows

5 represent fiber directions while yellow arrows indicate neurites. Quantification of the c) neurite

6 length for cortical neurons (at least 100 neurites from 5 scaffolds each group) and d) average

7 length of 10 longest neurites for DRG explants $(\mathrm{n}=5$ for control, $\mathrm{n}=6$ for CRISPRa-GDNF); $p$ $8<0.05, \stackrel{* *}{p} p<0.01$.

\section{4. Discussion}

11 Biodegradable polymers, such as PCL, are widely used for the fabrication of tissue 12 engineering scaffolds. The chemical and biological stability of PCL makes it difficult for surface 
1 modification. Therefore, simple noncovalent binding are methods usually accompanied by a

2 quick loss of the coating layers due to insufficient interfacial interactions. On the other hand, the

3 chemical modifications typically involve complex, laborious, and environmentally unfriendly

4 chemical synthesis. Utilizing the hydrophobicity of PCL, the SAP coating can alter the surface

5 charge of PCL fiber scaffolds. This approach offers a high level of customization as different

6 peptides can be designed and incorporated into the coating according to specific needs (eg:

7 surfaces with a positive charge, negative charge, cell adhesive peptides, etc). These

8 functionalized SAPs can be coated onto the fibers alone or as a cocktail, and the ratio of different

9 peptides can be adjusted easily. Therefore, the SAP coating is easy-to-implement and sufficiently

10 stable to perform more than one role (eg: immobilization of biomolecules on the surface and 11 support specific cell adhesion).

12 By altering the surface charge, the PCL@SAP group demonstrated improved cell adhesion 13 as compared to plain PCL (Fig. 3), similar to the effect of gas-plasma treatment on cell culture 14 dishes, which makes them more hydrophilic and negatively-charged [28]. We conjugated RGD 15 to the $\mathrm{SAP}^{+}$as the RGD peptide can be recognized by the integrin subsets and as such may 16 further enhance cell adhesion and spreading (Fig. 3) [29]. Moreover, the SAP backbone can also 17 be conjugated with other peptide sequences other than RGD, such as laminin-derived peptides 18 (IKVAV, YIGSR), N-cadherin mimetic peptide (HAVDI), and Wnt mimetic ligand (MDGECL), 19 thereby offering superb flexibility for various applications [30]. For example, the laminin20 derived peptides can be conjugated to enhance neuronal adhesion and neurite outgrowth for 21 neural tissue engineering. Therefore, we herein demonstrate a convenient method for the surface 22 modification of PCL fibers and a promising platform for the investigation of cell-substrate 23 interactions. 
2 viral manner, which is safer as compared to virus-mediated delivery that raises biosafety

3 concerns. While non-viral polyplexes usually cause some cytotoxicity issues, an optimized

4 delivery method can keep the toxicity under an acceptable level. Here, the cells grew with a high

5 proliferation rate and covered over $90 \%$ scaffold area within 3 days, suggesting that the cell

6 viability was acceptable. Such fiber scaffolds also provide more localized and sustained delivery

7 of the CRISPR/dCas9 system than existing methods. In particular, the scaffold-mediated delivery

8 approach ensures localized availability of drugs, which may reduce systemic side effects that is

9 often prominent in systemic delivery approaches. Previous in vivo studies showed that similar

10 approaches with scaffold-mediated gene delivery platforms locally released the complexes into

11 the tissues within $300 \mu \mathrm{m}$ from the edge of the scaffolds [31, 32]. Published results showed that

12 the complexes immobilized on the scaffolds were directly uptaken by the cells from the scaffold

13 surface $[14,18]$. Here, Cas9-GFP signals in cells were observed within two days, where the

14 cumulative release was only less than $5 \%$, suggesting that the cells also took up the complexes

15 directly from the scaffold surface. Although there were some larger polyplex aggregates on the

16 scaffold, cellular uptake was not affected as around $97.3 \pm 2.4 \%$ cells were found to contain the

17 labelled polyplexes within the cell body (Fig. S16). Furthermore, the modified nanofiber 18 scaffolds can also provide topographical cues for tissue regeneration. In addition to 19 CRISPR/dCas9 system, the coating is expected to be able to immobilize other biomolecules such 20 as nucleic acids (siRNA, miRNA, plasmids) and proteins on the scaffold surfaces.

21 In addition to the adsorption of CRISPR complexes on the SAP coated nanofibers, the 22 enhanced cellular uptake of the complexes can also be attributed to the increased rate of cell 23 proliferation. The transfection efficiency of the complexes might be affected by the rate of cell 
1 proliferation during which the cell membranes undergo disruption and reconstruction [33]. Here,

2 the U2OS cells proliferated most quickly on the PCL@SAP'@SAP+-RGD scaffold (Fig. S8),

3 thereby possibly leading to the enhanced uptake of pDNA/PEIpro complexes. However, we did

4 not achieve desirable transfection efficiency with hNPCs, a more realistic model, even by bolus

5 delivery (Fig. S14). One major limitation of this platform is that the transfection efficiency may

6 be affected by the effectiveness and efficiency of the delivery vehicles. Currently, low

7 transfection efficiency is a common problem when transfecting the difficult-to-transfect primary

8 cells, such as hNPCs, hMSCs and dermal fibroblasts [34, 35]. Although PEIpro was shortlisted

9 as the most efficient commercially available transfection reagent through our initial optimization 10 studies (Fig. S14), substituting PEIpro with a better-designed delivery vehicle may further 11 improve the gene delivery efficiency of the scaffold to the hNPCs and neural cells in general. In 12 addition, ribonucleoprotein may be a better format than pDNA in terms of CRISPR complex 13 delivery due to less off-target effects and higher efficiency. As delivery of CRISPR complexes to 14 primary neural cells, particularly post-mitotic neurons, may be challenging, a combination of the 15 improved format (such as ribonucleoprotein) and delivery vehicle will be needed to achieve 16 better results. Furthermore, a comprehensive in vivo evaluation of both transfection and gene 17 activation for tissue regeneration will be needed for future pre-clinical studies.

18 In this study, we only targeted a single gene (GDNF) as a proof of concept. By delivering 19 CRISPRa systems through SAP-coated nanofibers, GDNF expression was activated and hence 20 leading to the production of bioactive GDNF by cells, as demonstrated by the neurite outgrowth 21 assays. While the main objective of this study is to evaluate the ability of the coating to deliver 22 CRISPRa systems, the level of secreted GDNF $\left(1.0 \times 10^{-3} \mathrm{pg} / \mathrm{cell} / 24 \mathrm{~h}\right)$ is comparable to the 23 approaches that directly deliver GDNF transgene $\left(4.8 \times 10^{-4}\right.$ to $\left.1.7 \times 10^{-1} \mathrm{pg} / \mathrm{cell} / 24 \mathrm{~h}\right)$ and was 
1 effective in stimulating neurite outgrowth [36-40]. GDNF has already been well-established to

2 enhance neurite outgrowth of rat DRG explants and cortical neurons. Hence, we did not include

3 the GDNF-containing media as a positive control to save resources. In the literature, GDNF

4 added at a concentration range of 1 to $100 \mathrm{ng} / \mathrm{ml}$ induced 1.5 to 7 -folds increase in neurite length

5 of the rat DRG in a dose-dependent manner [41-44]. Similarly, neurite length of rat cortical

6 neurons increased 1.2 to 3 -folds in the presence of 0.1 to $100 \mathrm{ng} / \mathrm{ml} \mathrm{GDNF}$ in a dose-dependent

7 manner [45-47]. The increase in neurite length of DRG and cortical neurons in our experiment

8 were about 1.5 -folds and 2 -folds of the control respectively, which are within the range of the

9 published results. A small amount of GDNF as little as $70 \mathrm{pg} / \mathrm{ml}$ could stimulate neurite

10 outgrowth of spinal neurons [48].

11 In addition, the CRISPRa system is also capable of targeting multiple genes, requiring only

12 additional sgRNAs. Future works may extend the application of the CRISPRa systems-loaded 13 platform to relevant primary cells, such as glia and meningeal cells, which could be ideal targets 14 in vivo to secrete GDNF locally to enhance nerve regeneration. This ability of selective gene 15 upregulation provides a promising strategy to direct stem or progenitor cell differentiation for 16 regenerative medicine applications [49]. For example, these scaffolds are expected to be capable 17 of localized delivery of CRISPR/dCas9 to direct neuronal differentiation of stem cells through 18 the activation of transcription factors, which will contribute to neural regeneration as well [50, 19 51]. Besides the CRISPRa system, this scaffold-mediated CRISPR delivery system is also 20 suitable for CRISPR knockout (CRISPRko) and CRISPRi systems when the corresponding 21 sgRNAs and Cas9 or dCas9 expressing vectors are utilized. 


\section{5. Conclusion}

2 In summary, we synthesized two amphiphilic SAPs with opposite net charges, SAP $^{-}$and

3 SAP $^{+}$-RGD. The SAP was first coated onto PCL nanofibers through strong hydrophobic

4 interactions, and the pDNA/PEIpro complexes and SAP ${ }^{+}$-RGD were then easily absorbed via

5 electrostatic interactions. The SAP-coated scaffolds facilitated efficient loading and sustained

6 release of the pDNA complexes, while similar amount of pDNA complexes were completely

7 released from plain PCL scaffolds within 7 days. Meanwhile, the SAP coating effectively

8 enhanced cell adhesion and proliferation, even in the absence of serum proteins $(\mathrm{p}<0.0001)$.

9 Therefore, these scaffolds were utilized for the localized delivery of the CRISPR/dCas9 system,

10 which activated GDNF expression in mammalian cells and produced GDNF $\left(1.0 \times 10^{-3}\right.$

$11 \mathrm{pg} / \mathrm{cell} / 24 \mathrm{~h})$. The secreted GDNF retained its bioactivity and promoted neurite outgrowth, as

12 evidenced by the twice longer neurites than the non-treated groups. All these promising results

13 indicate that LbL SAP-coated nanofibers provide a useful tool for non-viral genome editing.

14 Together with the continuously developing CRISPR techniques, such scaffolds demonstrate 15 great potential in tissue regeneration.

\section{Competing interest}

18 The authors declare no conflict of interest.

\section{Acknowledgment}

21 This research is supported by the A*Star BMRC International Joint Grant - Singapore-China 22 Joint Research Program (Project no. 1610500024) and MOE AcRF Tier 1 Grant (Project No: 
1 RG148/14) awarded to SYC. The authors would like to thank Prof Hai-quan Mao (Johns

2 Hopkins University), Prof Kam Leong and Dr Hongxia Wang (Columbia University), Dr

3 Chuntao Liu (Sun Yat-sen University), Na Zhang, Junquan Lin, and William Ong (Nanyang

4 Technological University) for insightful suggestion and discussion, and Prof Sierin Lim

5 (Nanyang Technological University) for providing the E. Coli culture facilities.

\section{References}

[1] P.D. Hsu, E.S. Lander, F. Zhang, Development and applications of CRISPR-Cas9 for genome engineering, Cell 157(6) (2014) 1262-78.

[2] D.B. Cox, R.J. Platt, F. Zhang, Therapeutic genome editing: prospects and challenges, Nat Med 21(2) (2015) 121-31.

[3] P. Perez-Pinera, D.D. Kocak, C.M. Vockley, A.F. Adler, A.M. Kabadi, L.R. Polstein, P.I.

Thakore, K.A. Glass, D.G. Ousterout, K.W. Leong, F. Guilak, G.E. Crawford, T.E. Reddy, C.A. Gersbach, RNA-guided gene activation by CRISPR-Cas9-based transcription factors, Nat Methods 10(10) (2013) 973-6. [4] M.H. Hanewich-Hollatz, Z. Chen, L.M. Hochrein, J. Huang, N.A. Pierce, Conditional guide RNAs: programmable conditional regulation of CRISPR/Cas function in bacterial and mammalian cells via dynamic RNA nanotechnology, ACS central science 5(7) (2019) 12411249.

[5] M.F. La Russa, L.S. Qi, The New State of the Art: Cas9 for Gene Activation and Repression, Mol Cell Biol 35(22) (2015) 3800-9.

[6] M.H. Larson, L.A. Gilbert, X. Wang, W.A. Lim, J.S. Weissman, L.S. Qi, CRISPR interference (CRISPRi) for sequence-specific control of gene expression, Nature protocols $8(11)$ (2013) 2180.

[7] P. Perez-Pinera, D.D. Kocak, C.M. Vockley, A.F. Adler, A.M. Kabadi, L.R. Polstein, P.I. Thakore, K.A. Glass, D.G. Ousterout, K.W. Leong, RNA-guided gene activation by CRISPRCas9-based transcription factors, Nature methods 10(10) (2013) 973.

[8] S.M. Czerniecki, N.M. Cruz, J.L. Harder, R. Menon, J. Annis, E.A. Otto, R.E. Gulieva, L.V. Islas, Y.K. Kim, L.M. Tran, T.J. Martins, J.W. Pippin, H. Fu, M. Kretzler, S.J. Shankland, J. Himmelfarb, R.T. Moon, N. Paragas, B.S. Freedman, High-Throughput Screening Enhances Kidney Organoid Differentiation from Human Pluripotent Stem Cells and Enables Automated Multidimensional Phenotyping, Cell Stem Cell 22(6) (2018) 929-940 e4.

[9] H.X. Wang, M. Li, C.M. Lee, S. Chakraborty, H.W. Kim, G. Bao, K.W. Leong, CRISPR/Cas9-Based Genome Editing for Disease Modeling and Therapy: Challenges and Opportunities for Nonviral Delivery, Chem Rev 117(15) (2017) 9874-9906. [10] D.G. Tamay, T. Dursun Usal, A.S. Alagoz, D. Yucel, N. Hasirci, V. Hasirci, 3D and 4D Printing of Polymers for Tissue Engineering Applications, Front Bioeng Biotechnol 7 (2019) 164. 
[11] A. Cipitria, A. Skelton, T.R. Dargaville, P.D. Dalton, D.W. Hutmacher, Design, fabrication and characterization of PCL electrospun scaffolds - a review, Journal of Materials Chemistry 21(26) (2011) 9419-9453.

[12] H.J. Diao, W.C. Low, U. Milbreta, Q.R. Lu, S.Y. Chew, Nanofiber-mediated microRNA delivery to enhance differentiation and maturation of oligodendroglial precursor cells, J Control Release 208 (2015) 85-92.

[13] W.C. Low, P.O. Rujitanaroj, D.K. Lee, P.B. Messersmith, L.W. Stanton, E. Goh, S.Y. Chew, Nanofibrous scaffold-mediated REST knockdown to enhance neuronal differentiation of stem cells, Biomaterials 34(14) (2013) 3581-90.

[14] W.H. Chooi, W. Ong, A. Murray, J. Lin, D. Nizetic, S.Y. Chew, Scaffold mediated gene knockdown for neuronal differentiation of human neural progenitor cells, Biomater Sci 6(11) (2018) 3019-3029.

[15] J. Lin, D. Anopas, U. Milbreta, P.H. Lin, J.S. Chin, N. Zhang, S.K. Wee, A. Tow, W.T. Ang, S.Y. Chew, Regenerative rehabilitation: exploring the synergistic effects of rehabilitation and implantation of a bio-functional scaffold in enhancing nerve regeneration, Biomater Sci 7(12) (2019) 5150-5160.

[16] W. Ong, J. Lin, M.E. Bechler, K. Wang, M. Wang, C. Ffrench-Constant, S.Y. Chew, Microfiber drug/gene delivery platform for study of myelination, Acta Biomater 75 (2018) 152160.

[17] H.J. Diao, W.C. Low, Q.R. Lu, S.Y. Chew, Topographical effects on fiber-mediated microRNA delivery to control oligodendroglial precursor cells development, Biomaterials 70 (2015) 105-14.

[18] J.S. Chin, W.H. Chooi, H. Wang, W. Ong, K.W. Leong, S.Y. Chew, Scaffold-mediated nonviral delivery platform for CRISPR/Cas9-based genome editing, Acta Biomater 90 (2019) 60-70. [19] S. Jo, S.M. Kang, S.A. Park, W.D. Kim, J. Kwak, H. Lee, Enhanced adhesion of preosteoblasts inside 3D PCL scaffolds by polydopamine coating and mineralization, Macromol Biosci 13(10) (2013) 1389-95.

[20] L. Li, S. Hu, X. Chen, Non-viral delivery systems for CRISPR/Cas9-based genome editing: Challenges and opportunities, Biomaterials 171 (2018) 207-218.

[21] F.A. Ran, P.D. Hsu, J. Wright, V. Agarwala, D.A. Scott, F. Zhang, Genome engineering using the CRISPR-Cas9 system, Nat Protoc 8(11) (2013) 2281-2308.

[22] A. Chavez, J. Scheiman, S. Vora, B.W. Pruitt, M. Tuttle, P.R.I. E, S. Lin, S. Kiani, C.D. Guzman, D.J. Wiegand, D. Ter-Ovanesyan, J.L. Braff, N. Davidsohn, B.E. Housden, N. Perrimon, R. Weiss, J. Aach, J.J. Collins, G.M. Church, Highly efficient Cas9-mediated transcriptional programming, Nat Methods 12(4) (2015) 326-8.

[23] A. Murray, A. Letourneau, C. Canzonetta, E. Stathaki, S. Gimelli, F. Sloan-Bena, R. Abrehart, P. Goh, S. Lim, C. Baldo, F. Dagna-Bricarelli, S. Hannan, M. Mortensen, D. Ballard, D. Syndercombe Court, N. Fusaki, M. Hasegawa, T.G. Smart, C. Bishop, S.E. Antonarakis, J. Groet, D. Nizetic, Brief report: isogenic induced pluripotent stem cell lines from an adult with mosaic down syndrome model accelerated neuronal ageing and neurodegeneration, Stem Cells 33(6) (2015) 2077-84. [24] N. Zhang, U. Milbreta, J.S. Chin, C. Pinese, J. Lin, H. Shirahama, W. Jiang, H. Liu, R. Mi, A. Hoke, W. Wu, S.Y. Chew, Biomimicking Fiber Scaffold as an Effective In Vitro and In Vivo MicroRNA Screening Platform for Directing Tissue Regeneration, Adv Sci (Weinh) 6(9) (2019) 1800808 . 
[25] L. Delafosse, P. Xu, Y. Durocher, Comparative study of polyethylenimines for transient gene expression in mammalian HEK293 and CHO cells, J Biotechnol 227 (2016) 103-111. [26] S. Konermann, M.D. Brigham, A.E. Trevino, J. Joung, O.O. Abudayyeh, C. Barcena, P.D. Hsu, N. Habib, J.S. Gootenberg, H. Nishimasu, O. Nureki, F. Zhang, Genome-scale transcriptional activation by an engineered CRISPR-Cas9 complex, Nature 517(7536) (2015) 583-8.

[27] M.L. Maeder, S.J. Linder, V.M. Cascio, Y. Fu, Q.H. Ho, J.K. Joung, CRISPR RNA-guided activation of endogenous human genes, Nat Methods 10(10) (2013) 977-9.

[28] M.J. Lerman, J. Lembong, S. Muramoto, G. Gillen, J.P. Fisher, The Evolution of Polystyrene as a Cell Culture Material, Tissue Eng Part B Rev 24(5) (2018) 359-372.

[29] E. Ruoslahti, RGD and other recognition sequences for integrins, Annu Rev Cell Dev Biol 12 (1996) 697-715.

[30] N. Huettner, T.R. Dargaville, A. Forget, Discovering Cell-Adhesion Peptides in Tissue Engineering: Beyond RGD, Trends Biotechnol 36(4) (2018) 372-383.

[31] L.H. Nguyen, M. Gao, J. Lin, W. Wu, J. Wang, S.Y. Chew, Three-dimensional aligned nanofibers-hydrogel scaffold for controlled non-viral drug/gene delivery to direct axon regeneration in spinal cord injury treatment, Sci Rep 7 (2017) 42212.

[32] C. Pinese, J. Lin, U. Milbreta, M. Li, Y. Wang, K.W. Leong, S.Y. Chew, Sustained delivery of siRNA/mesoporous silica nanoparticle complexes from nanofiber scaffolds for long-term gene silencing, Acta Biomater 76 (2018) 164-177.

[33] S. Chernousova, M. Epple, Live-cell imaging to compare the transfection and gene silencing efficiency of calcium phosphate nanoparticles and a liposomal transfection agent, Gene Ther 24(5) (2017) 282-289.

[34] Y. Wang, L. Wang, Y. Zhu, J. Qin, Human brain organoid-on-a-chip to model prenatal nicotine exposure, Lab Chip 18(6) (2018) 851-860.

[35] O. Gresch, L. Altrogge, Transfection of difficult-to-transfect primary mammalian cells, Methods Mol Biol 801 (2012) 65-74.

[36] A. Sajadi, J.C. Bensadoun, B.L. Schneider, C. Lo Bianco, P. Aebischer, Transient striatal delivery of GDNF via encapsulated cells leads to sustained behavioral improvement in a bilateral model of Parkinson disease, Neurobiol Dis 22(1) (2006) 119-29.

[37] M. Suzuki, J. McHugh, C. Tork, B. Shelley, A. Hayes, I. Bellantuono, P. Aebischer, C.N. Svendsen, Direct muscle delivery of GDNF with human mesenchymal stem cells improves motor neuron survival and function in a rat model of familial ALS, Mol Ther 16(12) (2008) 2002-10.

[38] M. Suzuki, J. McHugh, C. Tork, B. Shelley, S.M. Klein, P. Aebischer, C.N. Svendsen, GDNF secreting human neural progenitor cells protect dying motor neurons, but not their projection to muscle, in a rat model of familial ALS, PLoS One 2(8) (2007) e689. [39] A.A. Akhtar, G. Gowing, N. Kobritz, S.E. Savinoff, L. Garcia, D. Saxon, N. Cho, G. Kim, C.M. Tom, H. Park, G. Lawless, B.C. Shelley, V.B. Mattis, J.J. Breunig, C.N. Svendsen, Inducible Expression of GDNF in Transplanted iPSC-Derived Neural Progenitor Cells, Stem Cell Reports 10(6) (2018) 1696-1704.

42 [40] A. Bakshi, S. Shimizu, C.A. Keck, S. Cho, D.G. LeBold, D. Morales, E. Arenas, E.Y. 43 Snyder, D.J. Watson, T.K. McIntosh, Neural progenitor cells engineered to secrete GDNF show 44 enhanced survival, neuronal differentiation and improve cognitive function following traumatic 45 brain injury, Eur J Neurosci 23(8) (2006) 2119-34. 
[41] C. Deister, C.E. Schmidt, Optimizing neurotrophic factor combinations for neurite outgrowth, J Neural Eng 3(2) (2006) 172-9.

[42] D. Santos, F. Gonzalez-Perez, X. Navarro, J. Del Valle, Dose-Dependent Differential Effect of Neurotrophic Factors on In Vitro and In Vivo Regeneration of Motor and Sensory Neurons, Neural Plast 2016 (2016) 4969523. [43] S. Takaku, H. Yanagisawa, K. Watabe, H. Horie, T. Kadoya, K. Sakumi, Y. Nakabeppu, F. Poirier, K. Sango, GDNF promotes neurite outgrowth and upregulates galectin-1 through the RET/PI3K signaling in cultured adult rat dorsal root ganglion neurons, Neurochem Int 62(3) (2013) 330-9.

[44] W.A. Lackington, Z. Koci, T. Alekseeva, A.J. Hibbitts, S.L. Kneafsey, G. Chen, F.J. O'Brien, Controlling the dose-dependent, synergistic and temporal effects of NGF and GDNF by encapsulation in PLGA microparticles for use in nerve guidance conduits for the repair of large peripheral nerve defects, J Control Release 304 (2019) 51-64. [45] R. Pellitteri, A. Russo, S. Stanzani, D. Zaccheo, Olfactory ensheathing cells protect cortical neuron cultures exposed to hypoxia, CNS Neurol Disord Drug Targets 14(1) (2015) 68-76. [46] J. Nielsen, K. Gotfryd, S. Li, N. Kulahin, V. Soroka, K.K. Rasmussen, E. Bock, V. Berezin, Role of glial cell line-derived neurotrophic factor (GDNF)-neural cell adhesion molecule (NCAM) interactions in induction of neurite outgrowth and identification of a binding site for NCAM in the heel region of GDNF, J Neurosci 29(36) (2009) 11360-76.

[47] C. Tohda, E. Joyashiki, Sominone enhances neurite outgrowth and spatial memory mediated by the neurotrophic factor receptor, RET, Br J Pharmacol 157(8) (2009) 1427-40.

[48] W. Gu, F. Zhang, Q. Xue, Z. Ma, P. Lu, B. Yu, Bone mesenchymal stromal cells stimulate neurite outgrowth of spinal neurons by secreting neurotrophic factors, Neurol Res 34(2) (2012) $172-80$.

[49] Z. Zhang, Y. Zhang, F. Gao, S. Han, K.S. Cheah, H.F. Tse, Q. Lian, CRISPR/Cas9 Genome-Editing System in Human Stem Cells: Current Status and Future Prospects, Mol Ther Nucleic Acids 9 (2017) 230-241. [50] J. Shao, M. Wang, G. Yu, S. Zhu, Y. Yu, B.C. Heng, J. Wu, H. Ye, Synthetic far-red lightmediated CRISPR-dCas9 device for inducing functional neuronal differentiation, Proc Natl Acad Sci U S A 115(29) (2018) E6722-E6730. [51] J.B. Black, A.F. Adler, H.G. Wang, A.M. D'Ippolito, H.A. Hutchinson, T.E. Reddy, G.S. Pitt, K.W. Leong, C.A. Gersbach, Targeted Epigenetic Remodeling of Endogenous Loci by CRISPR/Cas9-Based Transcriptional Activators Directly Converts Fibroblasts to Neuronal Cells, Cell Stem Cell 19(3) (2016) 406-14. 


\section{Graphical abstract}

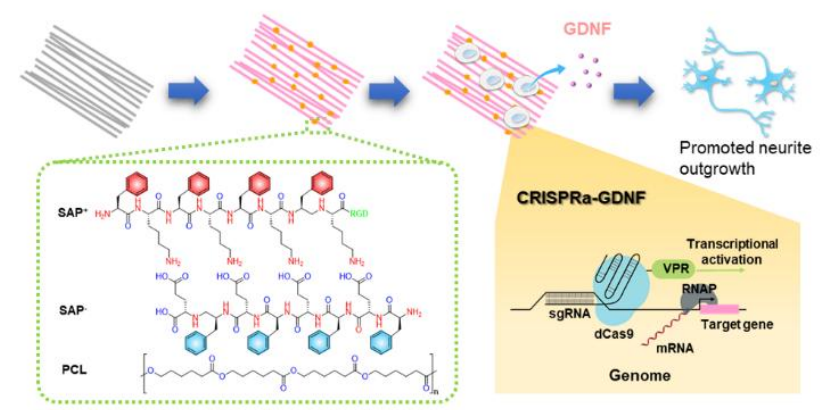

2

19

20

21

22

23

24

25

26

27

28

29

30

31

32

33

34

35

36

37

38

39

40

41

42

43

44

45

46

47

48

49

50

51

52

53

54

55

56

57

58

59

60

61

62

63 
Supplementary Information
Click here to download Supplementary Files: Revised Supplementary Information.DOCX

S. Revised Supplementary Information.DOCX

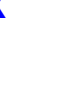
(1) 
Manuscript with changes tracked

Click here to download Supplementary Files: Revised manuscript_changes marked.DOCX

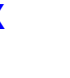

(1)

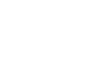

.

$\sqrt{2}$

upplementary Files: Revised manuscript_changes marked.DOCX

(1)

(1) 
Supplementary Information with changes tracked
Click here to download Supplementary Files: Revised Supplementary Information_changes marked.DOCX 


\section{${ }^{*}$ Declaration of Interest Statement}

\section{Declaration of interests}

$\bigotimes$ The authors declare that they have no known competing financial interests or personal relationships that could have appeared to influence the work reported in this paper.

$\square$ The authors declare the following financial interests/personal relationships which may be considered as potential competing interests:

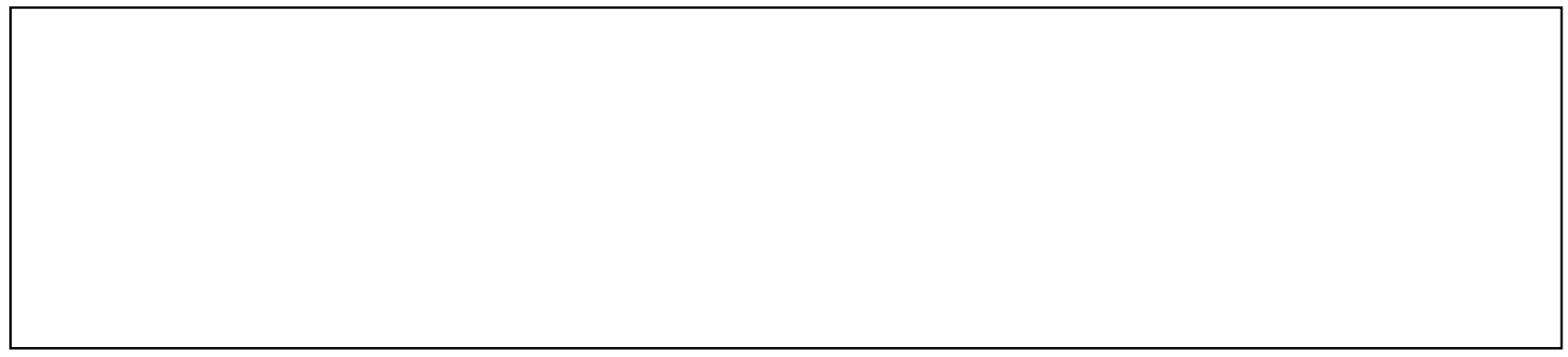

